\title{
Superposition operators and functions of bounded $p$-variation
}

\section{Gérard Bourdaud, Massimo Lanza de Cristoforis, Winfried Sickel}

\begin{abstract}
We characterize the set of all functions $f$ of $\mathbb{R}$ to itself such that the associated superposition operator $T_{f}: g \rightarrow f \circ g$ maps the class $B V_{p}^{1}(\mathbb{R})$ into itself. Here $B V_{p}^{1}(\mathbb{R}), 1 \leq p<\infty$, denotes the set of primitives of functions of bounded $p$-variation, endowed with a suitable norm. It turns out that such an operator is always bounded and sublinear. Also, consequences for the boundedness of superposition operators defined on Besov spaces $B_{p, q}^{s}\left(\mathbb{R}^{n}\right)$ are discussed.
\end{abstract}

\section{Introduction}

If $f$ is a function defined on the real line, the superposition operator associated with $f$ (sometimes also called composition operator) is defined by $T_{f}(g):=f \circ g$. In general, $T_{f}$ is a nonlinear operator. The Superposition Operator Problem (S.O.P.) for a given function space $E$ consists in finding the set $S(E)$ of all functions $f$ such that $T_{f}(E) \subseteq E$. The set $S(E)$ has been characterized only in a very limited number of cases, (cf. e.g., Appell and Zabrejko [1], [6], and [25, chap. 5].) In addition to the acting property of $T_{f}$, also the regularity of $T_{f}$ turns out to be of interest. So boundedness, continuity and differentiability of $T_{f}$ are properties which have been commonly investigated. In this paper, we concentrate on the boundedness properties of $T_{f}$. In particular, we shall analyze the inequalities describing the boundedness of $T_{f}$ in a qualitative way. We now turn to explain a specific issue in this direction. Clearly, under reasonable conditions on $f$ and $g$, we have

$$
\begin{aligned}
(f \circ g)^{\prime} & =\left(f^{\prime} \circ g\right) g^{\prime} \\
(f \circ g)^{\prime \prime} & =\left(f^{\prime \prime} \circ g\right)\left(g^{\prime}\right)^{2}+\left(f^{\prime} \circ g\right) g^{\prime \prime} .
\end{aligned}
$$

2000 Mathematics Subject Classification: 46E35, 47H30.

Keywords: Functions of bounded p-variation, homogeneous and inhomogeneous Besov spaces, Peetre's embedding theorem, boundedness of superposition operators. 
Then if $E_{1}$ is a function space defined by properties involving only derivatives up to the first order, such as the Sobolev space $W_{p}^{1}(\mathbb{R})$, we would expect the validity of an inequality of the type

$$
\left\|T_{f}(g)\right\|_{E_{1}} \leq c_{f}\left(1+\|g\|_{E_{1}}\right) \quad \forall g \in E_{1},
$$

for a suitable constant $c_{f}>0$. Instead, if $E_{2}$ is a function space defined by properties involving also second order derivatives, such as the Sobolev space $W_{p}^{2}(\mathbb{R})$, then we would expect the validity of an inequality of the type

$$
\left\|T_{f}(g)\right\|_{E_{2}} \leq c_{f}\left(1+\|g\|_{E_{2}}^{2}\right) \quad \forall g \in E_{2} .
$$

We say that an operator $T$ of a normed space $E$ to itself is sublinear, provided that there exists a constant $c$ such that

$$
\|T(g)\|_{E} \leq c\left(1+\|g\|_{E}\right) \quad \forall g \in E .
$$

Thus for example, superposition operators associated with Lipschitz continuous functions are sublinear on $W_{p}^{1}\left(\mathbb{R}^{n}\right)$ (cf. Marcus and Mizel [21]), while in case of $E=W_{p}^{2}\left(\mathbb{R}^{n}\right)$ inequality (1.1) holds (cf. [5] and [25, 5.2.4].)

Superposition operators associated with affine functions are trivially sublinear. Then a natural question occurs. Namely, do there exist nontrivial sublinear superposition operators in a given function space E? For Sobolev spaces $W_{p}^{m}\left(\mathbb{R}^{n}\right), m \in \mathbb{N}, 1 \leq p \leq \infty$, the answer is known. Such superposition operators exist if and only if $m=0$, or $m=1$, or $m=2$ and $p=1$ (cf. [5] and Proposition 1 below). Thus inequality (1.1) can be improved in $W_{1}^{2}\left(\mathbb{R}^{n}\right)$ but not in $W_{p}^{2}\left(\mathbb{R}^{n}\right)$ for $p>1$. A good way to explain the atypical behaviour of $W_{1}^{2}\left(\mathbb{R}^{n}\right)$ is to switch to function spaces of fractional order of smoothness such as the Besov spaces $B_{p, q}^{s}\left(\mathbb{R}^{n}\right)$ (see Section 4 for the definition). Thus $s=1+(1 / p)$ reveals to be the critical value for the sublinearity of superposition operators in Besov spaces. More precisely, we have the following.

Proposition 1 Let $1<p \leq+\infty$, and $s>1+(1 / p)$. Let $N$ be a norm on $\mathcal{D}\left(\mathbb{R}^{n}\right)$. Let $E$ be a normed function space such that $\mathcal{D}\left(\mathbb{R}^{n}\right) \subseteq E \subseteq W_{1, \text { loc }}^{1}\left(\mathbb{R}^{n}\right)$ and such that there exists a positive constant $A$ such that

$$
\sup _{h \neq 0}|h|^{1-s}\left(\int_{\mathbb{R}^{n}}\left|\frac{\partial g}{\partial x_{i}}(x+h)-\frac{\partial g}{\partial x_{i}}(x)\right|^{p} d x\right)^{1 / p} \leq A\|g\|_{E} \quad \forall g \in E
$$

for all $i=1, \ldots, n$.

If there exist a continuously differentiable function $f: \mathbb{R} \rightarrow \mathbb{R}$ and $a$ constant $B>0$ such that $T_{f}$ maps $\mathcal{D}\left(\mathbb{R}^{n}\right)$ into $E$, and such that the inequality

$$
\|f \circ g\|_{E} \leq B(N(g)+1) \quad \forall g \in \mathcal{D}\left(\mathbb{R}^{n}\right)
$$

holds, then $f$ must be an affine function. 
For $t>1+1 / p$, it is immediate to verify that Besov spaces $B_{p, q}^{t}\left(\mathbb{R}^{n}\right)$ satisfy condition $(1.2)$ with $1+(1 / p)<s<\min (t, 2)$. For a proof of Proposition 1, we refer the reader to Subsection 5.1, where a proof is provided under more general assumptions. Also, in Subsection 5.1 we provide further nontrivial examples of function spaces which fail to have sublinear superposition operators.

Of course, in Proposition 1 only a necessary condition is given. Looking back in history, most attention has been paid to the example $f(t)=|t|$, and to the associated superposition operator. Bourdaud and Meyer [9] and Oswald [22] have proved independently, and by exploiting different arguments, that the following holds. If $1 \leq p, q \leq \infty$ and $0<s<1+(1 / p)$, then there exists a constant $c>0$ such that

$$
\||g|\|_{B_{p, q}^{s}\left(\mathbb{R}^{n}\right)} \leq c\|g\|_{B_{p, q}^{s}\left(\mathbb{R}^{n}\right)} \quad \forall g \in B_{p, q}^{s}\left(\mathbb{R}^{n}\right) .
$$

Later, such statement has been generalized to superposition operators $T_{f}$ associated to functions $f$ such that the second derivative $f^{\prime \prime}$ is a finite measure ( cf. [6].) Combining Proposition 1 with such positive results, we understand that the limiting case $s=1+(1 / p)$ is still open. The following two negative results are well known (cf. [9] and Oswald [22].)

Proposition 2 There exists a function $g \in \mathcal{D}\left(\mathbb{R}^{n}\right)$ such that $|g| \notin B_{p, q}^{1+\frac{1}{p}}\left(\mathbb{R}^{n}\right)$, for all $p \in[1,+\infty]$ and $q \in[1,+\infty[$.

Thus we cannot hope that $T_{|.|}$maps $B_{p, q}^{1+\frac{1}{p}}\left(\mathbb{R}^{n}\right)$ to $B_{p, r}^{1+\frac{1}{p}}\left(\mathbb{R}^{n}\right)$ for some $p, q, r \in[1,+\infty]$, unless $r=+\infty$.

Proposition 3 Let $p \in[1,+\infty]$. There exists a function $g$ such that $g \in$ $B_{p, q}^{1+\frac{1}{p}}\left(\mathbb{R}^{n}\right)$, for all $\left.\left.q \in\right] 1,+\infty\right]$, and $|g| \notin B_{p, \infty}^{1+\frac{1}{p}}\left(\mathbb{R}^{n}\right)$.

Thus we can hope that $T_{|.|}$maps a Besov space $B_{p, q}^{1+\frac{1}{p}}\left(\mathbb{R}^{n}\right)$ with $p, q \in$ $[1,+\infty]$ to $B_{p, \infty}^{1+\frac{1}{p}}\left(\mathbb{R}^{n}\right)$ only if $q=1$. Indeed, such hope has been confirmed to be founded by Savaré [26] for $n=1$, who has proved the following result.

Proposition 4 For all $p \in[1,+\infty[$, there exists a constant $c$ such that

$$
\||g|\|_{B_{p, \infty}^{1+\frac{1}{p}}(\mathbb{R})} \leq c\|g\|_{B_{p, 1}^{1+\frac{1}{p}}(\mathbb{R})} \quad \forall g \in B_{p, 1}^{1+\frac{1}{p}}(\mathbb{R}) .
$$

As a matter of fact, Proposition 4 is just a corollary of the following stronger result of Savaré [26]. 
Theorem 1 For all $p \in\left[1,+\infty\left[\right.\right.$, there exists a Banach space $Z^{1+(1 / p), p}(\mathbb{R})$ such that the following continuous embeddings hold

$$
B_{p, 1}^{1+\frac{1}{p}}(\mathbb{R}) \hookrightarrow Z^{1+\frac{1}{p}, p}(\mathbb{R}) \hookrightarrow B_{p, \infty}^{1+\frac{1}{p}}(\mathbb{R}),
$$

and such that there exists a positive constant c satisfying

$$
\||g|\|_{Z^{1+(1 / p), p(\mathbb{R})}} \leq c\|g\|_{Z^{1+(1 / p), p}(\mathbb{R})} \quad \forall g \in Z^{1+(1 / p), p}(\mathbb{R}) .
$$

By Propositions 2 and 3, $Z^{1+(1 / p), p}(\mathbb{R})$ cannot be a Besov space. For $p=1$ it is the space of all functions $f \in W_{1}^{1}(\mathbb{R})$ such that $f^{\prime}$ is a function of bounded variation. For $1<p<\infty, Z^{1+(1 / p), p}(\mathbb{R})$ has been introduced by Tartar [28], using real interpolation (cf. Subsection 4.3.) As far as we know, no elementary description of the spaces $Z^{1+(1 / p), p}(\mathbb{R})$ for $p>1$ is available.

In the present paper, we consider the space $B V_{p}^{1}(\mathbb{R})$ of primitives of functions of bounded $p$-variation in the sense of Wiener. The space $B V_{p}^{1}(\mathbb{R})$ reveals to be a good substitute of $Z^{1+(1 / p), p}(\mathbb{R})$, for we can replace $Z^{1+(1 / p), p}(\mathbb{R})$ by $B V_{p}^{1}(\mathbb{R})$ in $(1.6)$. In this case, we have the following chain of continuous embeddings

$$
\dot{B}_{p, 1}^{1+\frac{1}{p}}(\mathbb{R}) \hookrightarrow B V_{p}^{1}(\mathbb{R}) \hookrightarrow \dot{B}_{p, \infty}^{1+\frac{1}{p}}(\mathbb{R})
$$

where $\dot{B}_{p, q}^{s}(\mathbb{R})$ denotes the homogeneous counterpart of the Besov space $B_{p, q}^{s}(\mathbb{R})$ (cf. Theorem 5). Furthermore, we will prove the following sharp result.

Theorem 2 Let $1 \leq p<\infty$. For all functions $f, g$ in $B V_{p}^{1}(\mathbb{R})$, the composite $f \circ g$ belongs to $B V_{p}^{1}(\mathbb{R})$ and

$$
\|f \circ g\|_{B V_{p}^{1}(\mathbb{R})} \leq\|f\|_{B V_{p}^{1}(\mathbb{R})}\left(1+2^{1 / p}\|g\|_{B V_{p}^{1}(\mathbb{R})}\right) .
$$

We note that in case $p=1$, Theorem 2 is due to Savaré and Tomarelli [27].

A few further remarks are in order. From the point of view of the S.O.P. the spaces $B V_{p}^{1}(\mathbb{R})$ have some extraordinary properties. They combine simplicity and beauty. It is a remarkable property that the composition of any pair of functions $f, g$ belonging to $B V_{p}^{1}(\mathbb{R})$ still remains in the same class. The corresponding property is not shared by the Besov spaces $B_{p, q}^{1+(1 / p)}(\mathbb{R})$, no matter what $q$ is. We do not know whether the same is true for the Tartar spaces. Also, Theorem 2 and the membership of the identity function in $B V_{p}^{1}(\mathbb{R})$ imply immediately that $S\left(B V_{p}^{1}(\mathbb{R})\right.$ ) coincides with $B V_{p}^{1}(\mathbb{R})$ (cf. Theorem 4.) 
Moreover, as explained above, the classes $B V_{p}^{1}(\mathbb{R})$ represent a limiting case for sublinear estimates. As the Tartar spaces, the classes $B V_{p}^{1}(\mathbb{R})$ can be used as a starting point to derive estimates for the superposition operator in Besov spaces both in the limiting case $s=1+(1 / p)$, and in the case $0<s<1+(1 / p)$. This explains why we have turned our attention to the S.O.P. in the classes $B V_{p}^{1}(\mathbb{R})$. For instance, by the embeddings (1.7) and by Theorem 2 , it follows that if $f \in B V_{p}^{1}(\mathbb{R})$ and $f(0)=0$, then $T_{f}$ maps $B_{p, 1}^{1+(1 / p)}(\mathbb{R})$ to $B_{p, \infty}^{1+(1 / p)}(\mathbb{R})$. Such result can be improved by replacing $B V_{p}^{1}(\mathbb{R})$ by the strictly larger class $U_{p}^{1}(\mathbb{R})$ introduced by Bourdaud and Kateb [8], in order to obtain superposition operators on Besov spaces of order $s<1+(1 / p)$ (cf. Theorems 7-10).

In a forthcoming paper we also investigate continuity and differentiability properties of the superposition operator in $B V_{p}^{1}(\mathbb{R})$.

\section{Contents of the paper}

In Section 2, we give the definition and basic properties of functions of bounded $p$-variation and their primitives. In Section 3, we prove Theorem 2 in a more precise form. In Section 4, we recall some basic facts on Besov spaces, with a special attention to the fundamental theorem of Peetre, which allows a comparison of the space $B V_{p}(\mathbb{R})$ of functions of bounded $p$-variation and the homogeneous Besov spaces of order $1 / p$. Section 5 contains new results on the superposition operator in Besov spaces of order $s \leq 1+(1 / p)$. In Section 6, we extend a part of our results to the $n$-dimensional setting.

\section{Notation}

We denote by $C(\mathbb{R})$ the set of continuous real valued functions on the real line. We denote by $C_{b}(\mathbb{R})$ the Banach space of the bounded functions of $C(\mathbb{R})$ endowed with the sup-norm, and by $C_{0}(\mathbb{R})$ the Banach subspace of the functions of $C_{b}(\mathbb{R})$ with 0 limiting value at infinity. We denote by $\mathcal{P}_{m}$ the set all polynomials of degree at most $m$. We denote by $\|\cdot\|_{p}$, with $p \in[1,+\infty]$ the standard $L^{p}$ norm. For any admissible couple of Banach spaces $\left(A_{0}, A_{1}\right)$ and for $\left.p \in[1,+\infty], t \in\right] 0,1\left[,\left(A_{0}, A_{1}\right)_{t, p}\right.$ denotes the LionsPeetre interpolation space (cf. [3, par. 3.1] or [29, 1.3.2]). Function spaces considered in this paper are distribution spaces, with the notable exceptions of $\mathcal{V}_{p}$ and $\mathcal{U}_{p}$. Thus in most cases the word "function" must be understood as "equivalence class of functions with respect to almost everywhere equality". We denote by $\mathbb{N}$ the set of nonnegative integers.

Acknowledgements. We thank Bernard Maurey for a discussion which led us to an important simplification in the statement and proof of Theorem 3. Also, we are indebted to Djalil Kateb, whose work on superposition operators was a source of inspiration and motivation for us. 


\section{Functions of bounded $p$-variation and their primi- tives}

\subsection{Functions of bounded $p$-variation}

Let $p \in\left[1,+\infty\left[\right.\right.$. Let $I$ be an interval of $\mathbb{R}$. We consider the space $\mathcal{V}_{p}(I)$ of functions of bounded $p$-variation introduced by Wiener [32]. A function $f: I \rightarrow \mathbb{R}$ belongs to $\mathcal{V}_{p}(I)$ if there exists $c>0$ such that

$$
\sum_{k=1}^{N}\left|f\left(t_{k}\right)-f\left(t_{k-1}\right)\right|^{p} \leq c^{p},
$$

for all finite sequences $t_{0}<t_{1}<\cdots<t_{N}$ in $I$. The infimum of such constants $c$ is denoted by $\nu_{p}(f, I)$. We note that in the above definition, we could as well take $t_{0} \leq t_{1} \leq \cdots \leq t_{N}$, without effecting $\nu_{p}(f, I)$. We use the abreviations $\mathcal{V}_{p}:=\mathcal{V}_{p}(\mathbb{R})$ and $\nu_{p}(f):=\nu_{p}(f, \mathbb{R})$. By considering a finite sequence with only two terms, we obtain

$$
|f(x)-f(y)| \leq \nu_{p}(f, I),
$$

for all $x, y \in I$. Hence, every element of $\mathcal{V}_{p}(I)$ is a bounded function. One can easily prove that $\mathcal{V}_{p}(I)$ becomes a Banach space if endowed with the following norm

$$
\|f\|_{\mathcal{V}_{p}(I)}:=\sup _{x \in I}|f(x)|+\nu_{p}(f, I) .
$$

According to Wiener [32], the right limit of $f \in \mathcal{V}_{p}(I)$ exists at each point of $I$ which is not the right endpoint. Moreover,

$$
\sum_{k=1}^{N}\left|f\left(t_{k}+\right)-f\left(t_{k-1}+\right)\right|^{p} \leq \nu_{p}(f, I)^{p},
$$

for all finite sequences $t_{0}<t_{1}<\cdots<t_{N}$ in $I$, such that $t_{N}$ is not the right endpoint of $I$, and a corresponding property holds for left limits. We complete the family $\mathcal{V}_{p}$ by defining $\mathcal{V}_{\infty}(I)$ as the set of bounded functions of $I$ to $\mathbb{R}$, having discontinuities only of the first kind. Then $\mathcal{V}_{\infty}(I)$ is a Banach space for the norm

$$
\nu_{\infty}(f, I):=\sup _{x \in I}|f(x)| .
$$

Definition 1 Let $f: \mathbb{R} \rightarrow \mathbb{R}$ be a function having discontinuities only of the first kind. Then $f$ is said to be normalized if

$$
f(x)=\frac{1}{2}(f(x+)+f(x-))
$$

for all $x$ in $\mathbb{R}$. 
Now we have the following results, which are of straightforward verification.

Proposition 5 Let $f: \mathbb{R} \rightarrow \mathbb{R}$ be a normalized function. If $f$ vanishes almost everywhere, then $f$ vanishes everywhere. Moreover, we have $f \in$ $L^{\infty}(\mathbb{R})$ if and only if $f$ is bounded. If $f$ is bounded, then

$$
\|f\|_{\infty}=\sup _{x \in \mathbb{R}}|f(x)|
$$

Proposition 6 Let $p \in[1,+\infty]$ and $f \in \mathcal{V}_{p}$. Let $\tilde{f}: \mathbb{R} \rightarrow \mathbb{R}$ be the function defined by

$$
\tilde{f}(x)=\frac{1}{2}(f(x+)+f(x-))
$$

for all $x \in \mathbb{R}$. Then the function $\tilde{f}$ is normalized, and belongs to $\mathcal{V}_{p}$, and satisfies the following inequality

$$
\nu_{p}(\tilde{f}) \leq \nu_{p}(f)
$$

Proposition 7 Let $f: \mathbb{R} \rightarrow \mathbb{R}$ be a measurable function. Then there exists at most one normalized function $\tilde{f}: \mathbb{R} \rightarrow \mathbb{R}$ such that $f=\tilde{f}$ almost everywhere in $\mathbb{R}$. If $\tilde{f}$ exists and if $g: \mathbb{R} \rightarrow \mathbb{R}$ is a measurable function equal to $f$ almost everywhere, then $\tilde{g}$ exists and is equal to $\tilde{f}$.

For further properties of functions of bounded $p$-variation, we refer to the comprehensive study of Bruneau [11].

\subsection{Functions of bounded $p$-variation as distributions}

Since $\mathcal{V}_{p}$ is evidently not a space of distributions, it is sometimes desirable to replace it by a space of distributions having similar properties. For $p=1$ we refer to Federer [14], Giusti [18] and Savaré [26].

Definition 2 Let $p \in[1,+\infty]$. We denote by $\mathcal{B} \mathcal{V}_{p}(\mathbb{R})$ the set of functions $f: \mathbb{R} \rightarrow \mathbb{R}$ such that there exists a function $g \in \mathcal{V}_{p}$ which coincides with $f$ almost everywhere, and we set

$$
\varepsilon_{p}(f):=\inf \left\{\nu_{p}(g): \quad g \in \mathcal{V}_{p}, g=f \text { a.e. }\right\} \quad \forall f \in \mathcal{B V}_{p}(\mathbb{R}) .
$$

We denote by $B V_{p}(\mathbb{R})$ the quotient set of $\mathcal{B} \mathcal{V}_{p}(\mathbb{R})$ modulo equality almost everywhere. If $h \in B V_{p}(\mathbb{R})$, we still denote by $\varepsilon_{p}(h)$, the number $\varepsilon_{p}(f)$, for any of the representatives $f$ of $h$. 
Remark. As customary, we denote by $B V(\mathbb{R})$ the set of all $f \in L_{\ell o c}^{1}(\mathbb{R})$ such that

$$
\sup \left\{\int_{-\infty}^{\infty} f(x) \Phi^{\prime}(x) d x: \quad \Phi \in \mathcal{D}(\mathbb{R}),|\Phi(x)| \leq 1 \quad \text { for all } \quad x \in \mathbb{R}\right\}
$$

is finite or, equivalently, such that the distributional derivative of $f$ is a finite Borel measure. Then $B V_{1}(\mathbb{R})=B V(\mathbb{R})$ as sets and the number $\varepsilon_{1}(f)$ coincides with the expression (2.1) and with the total variation of the measure $f^{\prime}$ (cf. e.g. Giusti $[18$, p. 29]).

By Proposition 7, one can easily derive the following statement.

Proposition 8 Let $p \in[1,+\infty]$. If $f \in B V_{p}(\mathbb{R})$, then $f$ has a unique normalized representative $\tilde{f} \in \mathcal{V}_{p}$. Moreover, we have $\varepsilon_{p}(f)=\nu_{p}(\tilde{f})$.

From now on, we shall consider $B V_{p}(\mathbb{R})$ as a Banach space of distributions, endowed with the following norm

$$
\|f\|_{B V_{p}(\mathbb{R})}:=\varepsilon_{p}(f)+\|f\|_{\infty}=\nu_{p}(\tilde{f})+\sup _{x \in \mathbb{R}}|\tilde{f}(x)| \quad \forall f \in B V_{p}(\mathbb{R})
$$

if $p<\infty$, and

$$
\|f\|_{B V_{\infty}(\mathbb{R})}:=\|f\|_{\infty}=\sup _{x \in \mathbb{R}}|\tilde{f}(x)| \quad \forall f \in B V_{\infty}(\mathbb{R}) .
$$

\subsection{Primitives of functions of bounded $p$-variation}

Definition 3 Let $p \in[1,+\infty]$. We say that a function $f: \mathbb{R} \rightarrow \mathbb{R}$ belongs to $B V_{p}^{1}(\mathbb{R})$ if $f$ is Lipschitz continuous and if its distributional derivative belongs to $B V_{p}(\mathbb{R})$.

By classical properties of Lipschitz continuous functions and by Proposition 8 , we derive the following alternative definition of $B V_{p}^{1}(\mathbb{R})$.

Proposition 9 Let $p \in[1,+\infty]$. Then the following statements hold.

(i) If $h \in B V_{p}(\mathbb{R})$, and $\alpha \in \mathbb{R}$, then the function $f: \mathbb{R} \rightarrow \mathbb{R}$ defined by

$$
f(x):=\alpha+\int_{0}^{x} h(t) d t \quad \forall x \in \mathbb{R}
$$

belongs to $B V_{p}^{1}(\mathbb{R})$.

(ii) If $f \in B V_{p}^{1}(\mathbb{R})$, then there exists an unique normalized function $h \in$ $\mathcal{V}_{p}$, and a real number $\alpha$, such that (2.2) holds.

We endow $B V_{p}^{1}(\mathbb{R})$ with the norm

$$
\|f\|_{B V_{p}^{1}(\mathbb{R})}:=|f(0)|+\left\|f^{\prime}\right\|_{B V_{p}(\mathbb{R})} \quad \forall f \in B V_{p}^{1}(\mathbb{R}),
$$

which renders $B V_{p}^{1}(\mathbb{R})$ a Banach space. 


\section{Superposition in $B V_{p}^{1}$}

\subsection{A basic inequality}

In the following theorem, we introduce an inequality which is most helpful in estimating the derivative of the composition of two functions.

Theorem 3 Let $p \in[1, \infty[$. Then the inequalities

$$
\begin{aligned}
& \nu_{p}((f \circ g) h, I) \leq \nu_{p}(f, g(I))\left(\sup _{I}|h|+2^{1 / p} \nu_{p}(h, I)\right)+\nu_{p}(h, I) \sup _{g(I)}|f| \\
& \|(f \circ g) h\|_{\mathcal{V}_{p}(I)} \leq 2^{1 / p}\|f\|_{\mathcal{V}_{p}(g(I))}\|h\|_{\mathcal{V}_{p}(I)}
\end{aligned}
$$

where

$$
g(t):=\alpha+\int_{t_{0}}^{t} h(x) d x \quad \forall t \in I,
$$

hold for all intervals $I$ of $\mathbb{R}$, and for all $t_{0} \in I$, and for all $\alpha \in \mathbb{R}$, and for all functions $h \in \mathcal{V}_{p}(I)$, and for all functions $f \in \mathcal{V}_{p}(g(I))$.

Proof. We first prove inequality (3.1). Let $t_{0}<\cdots<t_{N}$ be points of $I$. Then we have

$$
\begin{aligned}
\left(\sum_{k=0}^{N-1} \mid f\left(g\left(t_{k+1}\right)\right) h(\right. & \left.\left.t_{k+1}\right)-\left.f\left(g\left(t_{k}\right)\right) h\left(t_{k}\right)\right|^{p}\right)^{1 / p} \leq \\
\leq & \left(\sum_{k=0}^{N-1}\left|f\left(g\left(t_{k+1}\right)\right)-f\left(g\left(t_{k}\right)\right)\right|^{p}\left|h\left(t_{k}\right)\right|^{p}\right)^{1 / p} \\
& +\left(\sum_{k=0}^{N-1}\left|f\left(g\left(t_{k+1}\right)\right)\right|^{p}\left|h\left(t_{k+1}\right)-h\left(t_{k}\right)\right|^{p}\right)^{1 / p}
\end{aligned}
$$

We now denote by $A_{1}^{1 / p}$ the first term in the right hand side of (3.3) and we note that the second term is less or equal to

$$
\left(\sup _{g(I)}|f|\right) \nu_{p}(h, I)
$$

We now turn to estimate $A_{1}$. We first split the set $\{0, \ldots, N\}$ of indexes into $J$ subsets of consecutive indexes on each of which the finite sequence $\left(g\left(t_{k}\right)\right)_{k=0, \ldots, N}$ is monotone. More precisely, it is easily seen that there exist integer numbers

$$
0=n_{0}<n_{1}<\cdots<n_{J}=N
$$

such that the following two conditions hold. 
(I) The restriction $s_{j}$ of the sequence $\left(g\left(t_{k}\right)\right)_{0 \leq k \leq N}$ to the set

$$
\left\{k \in \mathbb{N}: n_{j-1} \leq k \leq n_{j}\right\}
$$

is monotone for each index $j=1, \ldots, J$.

(II) The restriction of the sequence $\left(g\left(t_{k}\right)\right)_{0 \leq k \leq N}$ to the set

$$
\left\{k \in \mathbb{N}: n_{j-1} \leq k \leq n_{j}+1\right\}
$$

is not monotone for any of the indices $j=1, \ldots, J-1$.

Clearly,

$$
\begin{aligned}
A_{1}=\sum_{j=1}^{J-1} \sum_{k=n_{j-1}}^{n_{j}-1}\left|f\left(g\left(t_{k+1}\right)\right)-f\left(g\left(t_{k}\right)\right)\right|^{p}\left|h\left(t_{k}\right)\right|^{p} & \\
& +\sum_{k=n_{J-1}}^{n_{J}-1}\left|f\left(g\left(t_{k+1}\right)\right)-f\left(g\left(t_{k}\right)\right)\right|^{p}\left|h\left(t_{k}\right)\right|^{p}
\end{aligned}
$$

with the understanding that if $J=1$, then we omit the first term in the right hand side. We now assume that $J>1$, and we turn to estimate the first sum in the right hand side of (3.4), which we denote by $A_{2}$. For each $1 \leq j \leq J-1$, we define an integer $k_{j}$ such that

$$
n_{j-1} \leq k_{j}<n_{j} \quad \text { and } \quad\left|h\left(t_{k_{j}}\right)\right|=\max \left\{\left|h\left(t_{k}\right)\right|: n_{j-1} \leq k<n_{j}\right\} .
$$

Then we have

$$
A_{2} \leq \nu_{p}^{p}(f, g(I)) \sum_{j=1}^{J-1}\left|h\left(t_{k_{j}}\right)\right|^{p} .
$$

In order to estimate the right hand side of (3.5), we exploit the following lemma, which is of straightforward verification.

Lemma 1 Let $a, b, c \in \mathbb{R}, a<b<c$. Let $h$ be a measurable real valued function on $[a, c]$, such that

$$
\int_{a}^{b} h(x) d x \geq 0 \quad \text { and } \quad \int_{b}^{c} h(x) d x<0 .
$$

Then there exist numbers $u, v$ in $] a, c[$ such that $h(u) h(v) \leq 0$. 
We now claim that for all $j=1, \ldots, J-1$, there exists $\left.a_{j} \in\right] t_{k_{j}}, t_{n_{j}+1}[$ such that

$$
h\left(a_{j}\right) h\left(t_{k_{j}}\right) \leq 0 .
$$

The existence of $a_{j}$ is obvious if $h\left(t_{k_{j}}\right)=0$, or if $h$ vanishes at some $t \in] t_{k_{j}}, t_{n_{j}+1}[$. Otherwise, we apply Lemma 1 to the functions $\pm h$ in the interval $\left[t_{k_{j}}, t_{n_{j}+1}\right]$, and deduce the existence of $\left.a_{j}^{\prime}, a_{j}^{\prime \prime} \in\right] t_{k_{j}}, t_{n_{j}+1}[$ such that $h\left(a_{j}^{\prime}\right) h\left(a_{j}^{\prime \prime}\right)<0$. As we shall see in a moment, it is convenient to split the sum in (3.5), into a sum on the even terms, and into a sum on the odd terms. Thus we define $M$ to be the largest integer such that $2 M+1 \leq J$. Clearly,

$$
\sum_{j=1}^{J-1}\left|h\left(t_{k_{j}}\right)\right|^{p}= \begin{cases}\sum_{j=1}^{2 M}\left|h\left(t_{k_{j}}\right)\right|^{p} & \text { if } J-1 \text { is even, } \\ \sum_{j=1}^{2 M}\left|h\left(t_{k_{j}}\right)\right|^{p}+\left|h\left(t_{k_{J-1}}\right)\right|^{p} & \text { if } J-1 \text { is odd. }\end{cases}
$$

Then inequality (3.6) implies that

$$
\begin{aligned}
\sum_{j=1}^{2 M}\left|h\left(t_{k_{j}}\right)\right|^{p} & \leq \sum_{l=1}^{M}\left|h\left(t_{k_{2 l}}\right)-h\left(a_{2 l}\right)\right|^{p}+\sum_{l=1}^{M}\left|h\left(t_{k_{2 l-1}}\right)-h\left(a_{2 l-1}\right)\right|^{p} \\
\left|h\left(t_{k_{J-1}}\right)\right|^{p} & \leq\left|h\left(t_{k_{J-1}}\right)-h\left(a_{J-1}\right)\right|^{p} .
\end{aligned}
$$

By inequalities

$$
t_{n_{j-1}} \leq t_{k_{j}}<t_{n_{j}}, \quad t_{k_{j}}<a_{j}<t_{n_{j}+1}
$$

for $j=1, \ldots, J-1$, it follows that

$$
a_{j}<t_{n_{j}+1} \leq t_{n_{j+1}} \leq t_{k_{j+2}},
$$

for $J \geq 4$ and $j=1, \ldots, J-3$. Then the intervals $\left[t_{k_{2 l}}, a_{2 l}\right]$ are pairwise disjoint for $l=1, \ldots, M$. In the same way, the intervals $\left[t_{k_{2 l-1}}, a_{2 l-1}\right]$ are pairwise disjoint for $l=1, \ldots, M$, if $J$ is odd, and for $l=1, \ldots, M+1$, if $J$ is even. Thus we conclude that

$$
\sum_{j=1}^{J-1}\left|h\left(t_{k_{j}}\right)\right|^{p} \leq 2 \nu_{p}^{p}(h, I) .
$$

Then, by the monotonicity of $s_{J}$, by relations (3.3), (3.4), (3.5), (3.7), we conclude that

$$
\nu_{p}((f \circ g) h, I) \leq \nu_{p}(f, g(I))\left[2 \nu_{p}^{p}(h, I)+\sup _{I}|h|^{p}\right]^{1 / p}+\nu_{p}(h, I) \sup _{g(I)}|f|,
$$

and thus inequality (3.1) holds. Inequality (3.2) is an immediate consequence of inequality (3.1). 


\subsection{The main theorem on the S.O.P in $B V_{p}^{1}$}

By applying Theorem 3, we obtain the following more complete formulation of Theorem 2.

Theorem 4 Let $1 \leq p<\infty$. Then the following statements hold.

(i) If $f, g \in B V_{p}^{1}(\mathbb{R})$, then $f \circ g \in B V_{p}^{1}(\mathbb{R})$, and

$$
\|f \circ g\|_{B V_{p}^{1}(\mathbb{R})} \leq\|f\|_{B V_{p}^{1}(\mathbb{R})}\left(1+2^{1 / p}\|g\|_{B V_{p}^{1}(\mathbb{R})}\right) .
$$

(ii) Let $f: \mathbb{R} \rightarrow \mathbb{R}$ be a Borel measurable function. Then the operator $T_{f}$ maps $B V_{p}^{1}(\mathbb{R})$ to itself if and only if $f \in B V_{p}^{1}(\mathbb{R})$.

Proof. We first prove statement (i). By Rademacher's Theorem, and by De La Vallée Poussin's Theorem, we deduce that the distributional derivative of $f \circ g$ is $\left(f^{\prime} \circ g\right) g^{\prime}$, where $f^{\prime}, g^{\prime}$ denote the normalized representatives of the distributional derivatives of $f, g$, respectively. Moreover,

$$
(f \circ g)(x)=(f \circ g)(0)+\int_{0}^{x} f^{\prime}(g(t)) g^{\prime}(t) d t .
$$

Since $f^{\prime}, g^{\prime} \in \mathcal{V}_{p}$, then we can apply Theorem 3 , and conclude that $\left(f^{\prime} \circ g\right) g^{\prime} \in$ $\mathcal{V}_{p}$, and that $\left\|\left(f^{\prime} \circ g\right) g^{\prime}\right\|_{\mathcal{V}_{p}} \leq 2^{1 / p}\left\|f^{\prime}\right\|_{\mathcal{V}_{p}}\left\|g^{\prime}\right\|_{\mathcal{V}_{p}}$. Then $f \circ g \in B V_{p}^{1}(\mathbb{R})$, and statement (i) follows. We now prove statement (ii). Sufficiency is an immediate consequence of statement (i). For the necessity, we note that the identity map in $\mathbb{R}$ belongs to $B V_{p}^{1}(\mathbb{R})$. Then assumption $T_{f}\left(B V_{p}^{1}(\mathbb{R})\right) \subseteq$ $B V_{p}^{1}(\mathbb{R})$ implies $f \in B V_{p}^{1}(\mathbb{R})$.

Remark. Theorem 4 shows that for a superposition operator defined on $B V_{p}^{1}(\mathbb{R})$ the acting condition implies boundedness.

Example. We now consider the family $u_{\alpha}(x):=|x+\alpha|-|\alpha|$ for $x, \alpha \in \mathbb{R}$. A simple calculation yields $\left\|u_{\alpha}^{\prime}\right\|_{B V_{p}(\mathbb{R})}=3$, for $p \in[1,+\infty[$. Applying Theorem 4 , we obtain the estimate

$$
\||g+\alpha|-|\alpha|\|_{B V_{p}^{1}(\mathbb{R})} \leq c_{p}\|g\|_{B V_{p}^{1}(\mathbb{R})} \quad \forall g \in B V_{p}^{1}(\mathbb{R}),
$$

for a constant $c_{p}$ independent of $\alpha$. Such inequality, together with a standard argument (cf. e.g., [6]), can be used to deduce that for all $f \in B V_{1}^{1}(\mathbb{R})$ there exists a constant $c_{p, f}$ such that the following inequality holds

$$
\|f \circ g\|_{B V_{p}^{1}(\mathbb{R})} \leq c_{p, f}\left(1+\|g\|_{B V_{p}^{1}(\mathbb{R})}\right) \quad \forall g \in B V_{p}^{1}(\mathbb{R}) .
$$

Such argument has been employed by Savaré and Tomarelli [27, lem. 2.5] in case $p=1$. We note that our Theorem 4 is stronger. Indeed, $B V_{1}^{1}(\mathbb{R})$ is properly embedded in $B V_{p}^{1}(\mathbb{R})$, for $p>1$. 


\section{Besov spaces}

We now compare the spaces $\mathcal{V}_{p}$ and $B V_{p}$ with Besov spaces and with Tartar spaces, which have been used in the literature in connection with the S.O.P.. Since the norm of $\mathcal{V}_{p}$ is obviously dilation invariant, it is natural to compare $\mathcal{V}_{p}$ with the homogeneous Besov spaces $\dot{B}_{p, q}^{s}(\mathbb{R})$, which we now introduce, at least for the values of the exponents that we need in the sequel.

\subsection{Definition of Besov spaces}

Let $p, q \in[1,+\infty]$. If $s \in] 0,1\left[\right.$, then we denote by $\dot{B}_{p, q}^{s}\left(\mathbb{R}^{n}\right)$ the set of tempered distributions $f$ of $L_{\ell o c}^{p}\left(\mathbb{R}^{n}\right)$ such that

$$
\|f\|_{\dot{B}_{p, q}^{s}\left(\mathbb{R}^{n}\right)}:=\left(\int_{\mathbb{R}^{n}}\left(\frac{1}{|h|^{s}}\left(\int_{\mathbb{R}^{n}}|f(x+h)-f(x)|^{p} d x\right)^{1 / p}\right)^{q} \frac{d h}{|h|^{n}}\right)^{1 / q}<+\infty .
$$

We denote by $\dot{B}_{p, q}^{1}\left(\mathbb{R}^{n}\right)$ the set of tempered distributions $f$ of $L_{\ell o c}^{p}\left(\mathbb{R}^{n}\right)$ such that

$$
\begin{aligned}
\|f\|_{\dot{B}_{p, q}^{1}\left(\mathbb{R}^{n}\right)} & :=\left(\int_{\mathbb{R}^{n}}\left(\frac{1}{|h|}\left(\int_{\mathbb{R}^{n}}|f(x+h)+f(x-h)-2 f(x)|^{p} d x\right)^{1 / p}\right)^{q} \frac{d h}{|h|^{n}}\right)^{1 / q} \\
& <+\infty
\end{aligned}
$$

Both in case $s \in] 0,1[$, and in case $s=1$, usual modifications hold if $p=$ $+\infty$ or $q=+\infty$. If $s \in] 1,2]$, we denote by $\dot{B}_{p, q}^{s}\left(\mathbb{R}^{n}\right)$ the set of tempered distributions $f$ of $L_{\ell o c}^{p}\left(\mathbb{R}^{n}\right)$ such that $\partial_{j} f \in \dot{B}_{p, q}^{s-1}\left(\mathbb{R}^{n}\right)$, for all $j=1, \ldots, n$, and we set

$$
\|f\|_{\dot{B}_{p, q}^{s}\left(\mathbb{R}^{n}\right)}:=\sum_{j=1}^{n}\left\|\partial_{j} f\right\|_{\dot{B}_{p, q}^{s-1}\left(\mathbb{R}^{n}\right)} \quad \forall f \in \dot{B}_{p, q}^{s}\left(\mathbb{R}^{n}\right) .
$$

Clearly, $\|\cdot\|_{\dot{B}_{p, q}^{s}\left(\mathbb{R}^{n}\right)}$ is a seminorm on $\dot{B}_{p, q}^{s}\left(\mathbb{R}^{n}\right)$ for each $\left.\left.s \in\right] 0,2\right], p, q \in$ $[1,+\infty]$, and $\dot{B}_{p, q}^{s}\left(\mathbb{R}^{n}\right)$ is known as the homogeneous Besov space of indexes $s, p, q$. The seminorm $\|\cdot\|_{\dot{B}_{p, q}^{s}\left(\mathbb{R}^{n}\right)}$ enjoys remarkable properties of translation and dilation invariance. Indeed,

$$
\|f(\cdot-a)\|_{\dot{B}_{p, q}^{s}\left(\mathbb{R}^{n}\right)}=\lambda^{(n / p)-s}\|f(\lambda(\cdot))\|_{\dot{B}_{p, q}^{s}\left(\mathbb{R}^{n}\right)}=\|f\|_{\dot{B}_{p, q}^{s}\left(\mathbb{R}^{n}\right)},
$$

for all $a \in \mathbb{R}^{n}$ and for all $\lambda>0$. The kernel of the seminorm $\|\cdot\|_{\dot{B}_{p, q}^{s}\left(\mathbb{R}^{n}\right)}$ is easily seen to be $\mathcal{P}_{[s]}$. 
We now introduce the quotient space

$$
\dot{\tilde{B}}_{p, q}^{s}\left(\mathbb{R}^{n}\right):=\dot{B}_{p, q}^{s}\left(\mathbb{R}^{n}\right) / \mathcal{P}_{[s]} .
$$

As it is well known, $\dot{\tilde{B}}_{p, q}^{s}\left(\mathbb{R}^{n}\right)$ is a Banach space. We point out that often times in the literature, the space $\dot{\tilde{B}}_{p, q}^{s}\left(\mathbb{R}^{n}\right)$ is addressed to as the homogeneous Besov space, and what we have defined as $\dot{B}_{p, q}^{s}\left(\mathbb{R}^{n}\right)$ plays a secondary role. Instead, in this paper we will concentrate on the distribution space $\dot{B}_{p, q}^{s}\left(\mathbb{R}^{n}\right)$, which we address to as the homogeneous Besov space. The space $\dot{B}_{p, q}^{s}\left(\mathbb{R}^{n}\right)$ will be endowed with the seminorm $\|\cdot\|_{\dot{B}_{p, q}^{s}\left(\mathbb{R}^{n}\right)}$, except in some specific cases, such as $\dot{B}_{p, 1}^{1 / p}(\mathbb{R})$ (cf. (4.2)). Moreover, for our needs, it is important to keep the roles of $\dot{B}_{p, q}^{s}\left(\mathbb{R}^{n}\right)$ and of $\dot{\tilde{B}}_{p, q}^{s}\left(\mathbb{R}^{n}\right)$ distinct. In order to exploit known results on Besov spaces, and in particular the celebrated embedding Theorem of Peetre, we find convenient to note that $\dot{\tilde{B}}_{p, q}^{s}\left(\mathbb{R}^{n}\right)$ can be identified with a subspace of $\dot{B}_{p, q}^{s}\left(\mathbb{R}^{n}\right)$. Thus we introduce the following.

Proposition 10 (i) Let $p \in] 1,+\infty\left[\right.$. If $g$ is an element of $\dot{B}_{p, 1}^{1 / p}(\mathbb{R})$, then $g$ is a continuous function and the limit

$$
L_{g}:=\lim _{x \rightarrow \infty} g(x)
$$

exists in $\mathbb{R}$. In particular, each element of $\dot{B}_{p, 1}^{1 / p}(\mathbb{R})$ is congruent modulo $\mathcal{P}_{0}$ to exactly one element of $C_{0}(\mathbb{R})$. Moreover, the map of $\dot{B}_{p, 1}^{1 / p}(\mathbb{R})$ to $\left(C_{0}(\mathbb{R}),\|\cdot\|_{\infty}\right)$ which takes $g$ to $g-L_{g}$ is continuous.

(ii) If $g$ is an element of $\dot{B}_{1,1}^{1}(\mathbb{R})$, then $g$ is a continuous function and the limits

$$
L_{g}^{\prime}:=\lim _{x \rightarrow \infty} \frac{g(x)}{x}, \quad L_{g}^{\prime \prime}:=\lim _{x \rightarrow \infty}\left(g(x)-L_{g}^{\prime} x\right)
$$

exist in $\mathbb{R}$. In particular, each element of $\dot{B}_{1,1}^{1}(\mathbb{R})$ is congruent modulo $\mathcal{P}_{1}$ to exactly one element of $C_{0}(\mathbb{R})$. Moreover, the map of $\dot{B}_{1,1}^{1}(\mathbb{R})$ to $\left(C_{0}(\mathbb{R}),\|\cdot\|_{\infty}\right)$ which takes $g$ to the function $g-L_{g}^{\prime} \mathrm{id}_{\mathbb{R}}-L_{g}^{\prime \prime}$ is continuous. Here $\operatorname{id}_{\mathbb{R}}$ denotes the identity function in $\mathbb{R}$.

(iii) Let $p \in\left[1,+\infty\left[\right.\right.$. The space $\dot{B}_{p, 1}^{1 / p}(\mathbb{R}) \cap C_{0}(\mathbb{R})$ endowed with the restriction of the seminorm $\|\cdot\|_{\dot{B}_{p, 1}^{1 / p}(\mathbb{R})}$ is a Banach space isometric to $\dot{\tilde{B}}_{p, 1}^{1 / p}(\mathbb{R})$.

For a proof of Proposition 10, we refer to [4]. Let $p \in] 1,+\infty[$. By Proposition 10 , the space $\dot{B}_{p, 1}^{1 / p}(\mathbb{R})$ will be endowed with the norm

$$
g \mapsto\|g\|_{\dot{B}_{p, 1}^{1 / p}(\mathbb{R})}+\left|L_{g}\right| .
$$


We close this subsection by introducing the inhomogeneous Besov spaces.

Definition 4 Let $0<s \leq 2$ and $p, q \in[1,+\infty]$. The nonhomogeneous Besov space $B_{p, q}^{s}\left(\mathbb{R}^{n}\right)$ is the collection of all tempered distributions $f$ of $L^{p}\left(\mathbb{R}^{n}\right) \cap \dot{B}_{p, q}^{s}\left(\mathbb{R}^{n}\right)$.

As it is well known, $B_{p, q}^{s}\left(\mathbb{R}^{n}\right)$ endowed with the norm

$$
\|f\|_{B_{p, q}^{s}\left(\mathbb{R}^{n}\right)}:=\|f\|_{\dot{B}_{p, q}^{s}\left(\mathbb{R}^{n}\right)}+\|f\|_{p} \quad \forall f \in B_{p, q}^{s}\left(\mathbb{R}^{n}\right),
$$

is a Banach space.

\subsection{A function space related to $\dot{B}_{p, \infty}^{1 / p}(\mathbb{R})$}

In order to prove the main result of the next section, namely Theorem 7 , we resort to a class of functions $U_{p}^{1}(\mathbb{R})$ first introduced by Bourdaud and Kateb [8]. The elements of $U_{p}^{1}(\mathbb{R})$ will be primitives of functions which are bounded and belong to $\mathcal{U}_{p}(\mathbb{R})$, a function space we now turn to define.

Definition 5 Let $p \in[1,+\infty[$. Let $J$ be an interval of $\mathbb{R}$. We denote by $\mathcal{U}_{p}(J)$ the set of measurable functions $f: \mathbb{R} \mapsto \mathbb{R}$ such that the function $\sup _{|h| \leq t}|f(x+h)-f(x)|$ is measurable on $J$ in the variable $x$ for all $t>0$, and such that

$$
\|f\|_{\mathcal{U}_{p}(J)}^{p}:=\sup _{t>0} t^{-1} \int_{J} \sup _{|h| \leq t}|f(x+h)-f(x)|^{p} d x<+\infty .
$$

Just as we did to define $B V_{p}(\mathbb{R})$ starting from $\mathcal{V}_{p}(\mathbb{R})$ (cf. Definition 2), we now define $U_{p}(\mathbb{R})$ starting from $\mathcal{U}_{p}(\mathbb{R})$, by taking the functions which are almost everywhere equal to at least an element of $\mathcal{U}_{p}(\mathbb{R})$, and then by taking the quotient modulo equality almost everywhere. In particular, we endow $U_{p}(\mathbb{R})$ with the seminorm

$$
f \mapsto \inf \left\{\|g\|_{\mathcal{U}_{p}(\mathbb{R})}: g \in \mathcal{U}_{p}(\mathbb{R}), g=f \text { a.e. }\right\} .
$$

Then we introduce the following.

Definition 6 Let $p \in\left[1,+\infty\left[\right.\right.$. We denote by $U_{p}^{1}(\mathbb{R})$ the set of Lipschitz continuous functions $f: \mathbb{R} \rightarrow \mathbb{R}$ such that $f^{\prime}$ has a bounded Borel measurable representative of class $\mathcal{U}_{p}(\mathbb{R})$, and we set

$\|f\|_{U_{p}^{1}(\mathbb{R})}:=\inf \left\{\sup _{\mathbb{R}}|\Phi|+\|\Phi\|_{\mathcal{U}_{p}(\mathbb{R})}: \Phi\right.$ is a bounded Borel measurable function of class $\mathcal{U}_{p}(\mathbb{R}), \Phi=f^{\prime}$ a.e. $\} \quad \forall f \in U_{p}^{1}(\mathbb{R})$. 


\subsection{Peetre's theorem}

In his celebrated monograph on Besov spaces, J. Peetre [24] proves the validity of the following continuous embeddings

$$
\dot{\tilde{B}}_{p, 1}^{1 / p}(\mathbb{R}) \hookrightarrow \mathcal{V}_{p}(\mathbb{R}) \hookrightarrow \dot{\tilde{B}}_{p, \infty}^{1 / p}(\mathbb{R}),
$$

for $1 \leq p<\infty$. We wish to remark that $\dot{\tilde{B}}_{p, 1}^{1 / p}(\mathbb{R})$ and $\dot{\tilde{B}}_{p, \infty}^{1 / p}(\mathbb{R})$ are not spaces of distributions, but spaces of distributions modulo polynomials. Accordingly, the first embedding in (4.3) should be interpreted as a section, which associates to each equivalence class of $\dot{\tilde{B}}_{p, 1}^{1 / p}(\mathbb{R})$ a specific representative, while the second embedding in (4.3) should be interpreted as a quotient mapping, which associates to each function in $\mathcal{V}_{p}(\mathbb{R})$ an equivalence class modulo polynomials. Actually, we find convenient to introduce Peetre's Theorem in a slightly different version, by identifying $\dot{\tilde{B}}_{p, 1}^{1 / p}(\mathbb{R})$ with $\dot{B}_{p, 1}^{1 / p}(\mathbb{R}) \cap C_{0}(\mathbb{R})$ (cf. Proposition 10.)

Theorem 5 Let $p \in] 1,+\infty[$. Then the following chain of continuous embeddings hold

$$
\begin{array}{r}
\dot{B}_{p, 1}^{1 / p}(\mathbb{R}) \hookrightarrow\left(L^{\infty}(\mathbb{R}), B V_{1}(\mathbb{R})\right)_{1 / p, p}=\left(B V_{\infty}(\mathbb{R}), B V_{1}(\mathbb{R})\right)_{1 / p, p} \\
\hookrightarrow B V_{p}(\mathbb{R}) \hookrightarrow U_{p}(\mathbb{R}) \hookrightarrow \dot{B}_{p, \infty}^{1 / p}(\mathbb{R}) .
\end{array}
$$

Proof. The main ideas of the proof are explained in Peetre [24, thm. 7, p. 122]. For the definition of the Besov spaces of smoothness order 0 we refer to Peetre (loc. cit.) and Triebel [30], for properties of the real interpolation functor we refer to Bennet and Sharpley [2], Bergh and Löfström [3], Peetre (loc. cit.) and Triebel (loc. cit.), and for interpolation properties of Besov spaces to all of these books. We divide our proof into five parts.

1- We have the continuous embeddings

$$
\dot{B}_{p, 1}^{1 / p}(\mathbb{R}) \cap C_{0}(\mathbb{R}) \hookrightarrow\left(\dot{B}_{\infty, 1}^{0}(\mathbb{R}) \cap C_{b}(\mathbb{R}), \dot{B}_{1,1}^{1}(\mathbb{R}) \cap C_{0}(\mathbb{R})\right)_{1 / p, p},
$$

and

$$
\dot{B}_{1,1}^{1}(\mathbb{R}) \cap C_{0}(\mathbb{R}) \hookrightarrow B V_{1}(\mathbb{R}),
$$

(cf. e.g., Peetre [24, thm. 6, p. 106].) Hence, the first embedding is a consequence of Proposition 10, and of the fact that constant distributions are members of $L^{\infty}(\mathbb{R}) \cap B V_{1}(\mathbb{R})$.

2- We denote by $E$ the closure of $B V_{1}(\mathbb{R})$ in $L^{\infty}(\mathbb{R})$. By Bergh and Löfström [3, thm. 3.4.2(d)], we have

$$
\left(L^{\infty}(\mathbb{R}), B V_{1}(\mathbb{R})\right)_{1 / p, p}=\left(E, B V_{1}(\mathbb{R})\right)_{1 / p, p} .
$$


Hence, the second embedding is the consequence of the obvious embedding of $E$ into $B V_{\infty}(\mathbb{R})$.

3- We denote by $l_{N}^{p}$ the space $\mathbb{R}^{N}$, endowed with the $l^{p}$-norm. Let $t_{0}<$ $t_{1}<\cdots<t_{N}$ be a fixed finite sequence of real numbers. Then we consider the mapping

$$
\begin{array}{clc}
U: B V_{\infty}(\mathbb{R}) & \rightarrow & l_{N}^{\infty} \\
f & \mapsto\left(\tilde{f}\left(t_{k}\right)-\tilde{f}\left(t_{k-1}\right)\right)_{1 \leq k \leq N}
\end{array}
$$

We have

$$
\begin{aligned}
\|U(f)\|_{l_{N}^{\infty}} \leq 2\|f\|_{B V_{\infty}(\mathbb{R})} & \forall f \in B V_{\infty}(\mathbb{R}), \quad \text { and } \\
\|U(f)\|_{l_{N}^{1}} \leq\|f\|_{B V_{1}(\mathbb{R})} & \forall f \in B V_{1}(\mathbb{R}) .
\end{aligned}
$$

As it is known, the interpolation space $\left(L^{\infty}(X), L^{1}(X)\right)_{\frac{1}{p}, p}$ coincides with $L^{p}(X)$ as a set for all measure spaces $X$, and the corresponding norms are equivalent. Moreover, one can choose the equivalence constants for the norms so as to depend only on $p$, and not on the underlying measure space $X$, which in our case is the set $\left\{t_{0}, \ldots, t_{N}\right\}$ (cf. e.g., Bennet and Sharpley [2, lem. IV.4.5, p. 219, thm. V.1.6, p. 298].) Since the real interpolation provides an exact interpolation functor (cf. e.g., Bergh and Löfström [3, thm. 3.1.2]), there exists $c_{p}>0$ such that

$$
\|U(f)\|_{l_{N}^{p}} \leq c_{p}\|f\|_{\left(B V_{\infty}(\mathbb{R}), B V_{1}(\mathbb{R})\right)_{1 / p, p}} \quad \forall f \in\left(B V_{\infty}(\mathbb{R}), B V_{1}(\mathbb{R})\right)_{1 / p, p} .
$$

Taking the supremum for all sequences, we obtain

$$
\|f\|_{B V_{p}(\mathbb{R})} \leq c_{p}\|f\|_{\left(B V_{\infty}(\mathbb{R}), B V_{1}(\mathbb{R})\right)_{1 / p, p}} \quad \forall f \in\left(B V_{\infty}(\mathbb{R}), B V_{1}(\mathbb{R})\right)_{1 / p, p}
$$

for a possibly larger $c_{p}$.

4- We now prove the embedding $\mathcal{V}_{p}(\mathbb{R}) \hookrightarrow \mathcal{U}_{p}(\mathbb{R})$ by an argument of Marcinkiewicz $[20]$. Let $f \in \mathcal{V}_{p}(\mathbb{R})$ and let $t>0$. Then we have

$$
\begin{aligned}
\int_{\mathbb{R}|h| \leq t} \sup _{|h|}|f(x+h)-f(x)|^{p} d x & \\
= & \sum_{m \in \mathbb{Z}} \int_{m t}^{(m+1) t} \sup _{|h| \leq t}|f(x+h)-f(x)|^{p} d x \\
& =\int_{0}^{t} \sum_{m \in \mathbb{Z}} \sup _{|h| \leq t}|f(y+m t+h)-f(y+m t)|^{p} d y .
\end{aligned}
$$


Now let $\epsilon>0$ be fixed arbitrarily. For each $m \in \mathbb{Z}, y \in \mathbb{R}$, there exists $h_{m}(y) \in[-t, t]$ such that

$$
\begin{aligned}
\mid f\left(y+m t+h_{m}(y)\right)- & \left.f(y+m t)\right|^{p} \geq \\
& \geq \sup _{|h| \leq t}|f(y+m t+h)-f(y+m t)|^{p}-\frac{\epsilon}{4} 2^{-|m|} .
\end{aligned}
$$

Now the intervals of the family

$$
\{] y+2 l t, y+2 l t+h_{2 l}(y)[\}_{l \in \mathbb{Z}}
$$

are pairwise disjoint, and the same holds for

$$
\{] y+(2 l+1) t, y+(2 l+1) t+h_{2 l+1}(y)[\}_{l \in \mathbb{Z}} .
$$

Then we obtain

$$
\begin{aligned}
& \int_{0}^{t} \sum_{m \in \mathbb{Z}} \sup _{|h| \leq t} \mid f(y+m t+h)-\left.f(y+m t)\right|^{p} d y \leq \\
& \leq \int_{0}^{t} \sum_{m \in \mathbb{Z}}\left\{\left|f\left(y+m t+h_{m}(y)\right)-f(y+m t)\right|^{p}+\frac{\epsilon}{4} 2^{-|m|}\right\} d y \\
& \leq \int_{0}^{t}\left(2 \nu_{p}^{p}(f)+\epsilon\right) d y=t\left(2 \nu_{p}^{p}(f)+\epsilon\right) .
\end{aligned}
$$

Since $\epsilon>0$ is arbitrary, we obtain inequality

$$
\|f\|_{\mathcal{U}_{p}(\mathbb{R})} \leq 2^{1 / p} \nu_{p}(f) .
$$

5- The last embedding follows immediately from the definitions.

Remarks. 1- The interpolation space $\left(L^{\infty}(\mathbb{R}), B V_{1}(\mathbb{R})\right)_{1 / p, p}$ has been studied by Tartar [28], and then employed by Savaré [26] in order to prove Theorem 1. Indeed, the space considered by Savaré is defined as follows

$$
Z^{1+(1 / p), p}(\mathbb{R}):=\left\{f \in W_{p}^{1}(\mathbb{R}): f^{\prime} \in\left(L^{\infty}(\mathbb{R}), B V_{1}(\mathbb{R})\right)_{1 / p, p}\right\} .
$$

2- In the appendix, we prove the properness of the embeddings $B V_{p} \subset$ $U_{p} \subset \dot{B}_{p, \infty}^{1 / p}$ in case $p>1$. In case $p=1$, the first embedding turns out to be an equality. Indeed, if $f \in U_{1}(\mathbb{R})$, then the following holds

$$
\sup _{h \neq 0} \frac{1}{|h|} \int_{\mathbb{R}}|f(x+h)-f(x)| d x<+\infty,
$$

an inequality which is well known to imply that $f \in B V_{1}(\mathbb{R})$, (cf. e.g., DeVore and Lorentz [12, ch. 2, thm. 9.3].) 


\section{Superposition operators in Besov spaces}

We start with a proof of the necessity of the restriction $s \leq 1+(1 / p)$ for the existence of nontrivial sublinear superposition operators in Besov spaces (see Proposition 1.) Then we turn to a new superposition theorem in case $s=1+(1 / p)$ and we give conditions for the action of $T_{f}$ in Besov spaces of lower order of smoothness.

\subsection{Proof of Proposition 1}

As announced in the introduction, we shall prove here a slightly more sophisticated version of Proposition 1. In part for technical reasons, we split our considerations into two cases: case $p=1$ and case $p>1$.

Proposition 11 Let $p \in] 1,+\infty]$. Let $N$ be a norm on $\mathcal{D}\left(\mathbb{R}^{n}\right)$. Let $h: \mathbb{R} \rightarrow$ $[0,+\infty[$ be a function such that $h(t)>0$ if $t \in \mathbb{R} \backslash\{0\}$, and such that

$$
\lim _{t \rightarrow 0} h(t)|t|^{-1 / p}=0 .
$$

Let $E$ be a subspace of $W_{1, \text { loc }}^{1}\left(\mathbb{R}^{n}\right)$ containing $\mathcal{D}\left(\mathbb{R}^{n}\right)$ such that there exist a direction $\nu \in \mathbb{R}^{n},|\nu|=1$, and a positive constant $A$ such that

$$
\left(\int_{Q}\left|\frac{\partial g}{\partial \nu}(x+t \nu)-\frac{\partial g}{\partial \nu}(x)\right|^{p} d x\right)^{1 / p} \leq A h(t)\|g\|_{E}
$$

for all $g \in E$, for all cubes $Q \subset \mathbb{R}^{n}$ with $|Q|=1$, and for all $t \in[-1 / 2,1 / 2]$, where we understand that an obvious modification is applied to (5.1) and (5.2) when $p=+\infty$.

If there exist a continuously differentiable function $f: \mathbb{R} \rightarrow \mathbb{R}$ and a constant $B>0$ such that $T_{f}$ maps $\mathcal{D}\left(\mathbb{R}^{n}\right)$ into $E$, and such that the inequality (1.3) holds, then $f$ must be an affine function.

Proof. Without loss of generality, we may assume that $\nu=(1,0, \ldots, 0)$. Let $\varphi \in \mathcal{D}\left(\mathbb{R}^{n}\right)$ be such that $\varphi(x)=x_{1}$ on the cube $[-1,1]^{n}$. We now introduce the auxiliary functions

$$
g_{a}(x)=a \varphi(x) \quad \forall x \in \mathbb{R}^{n},
$$

for each $a \in] 1,+\infty\left[\right.$. Let $Q_{0}=[-1 / 2,1 / 2]^{n}$. Then

$$
\left(f \circ g_{a}\right)(x+t \nu)=f\left(a x_{1}+a t\right) \quad \forall(x, t) \in Q_{0} \times[-1 / 2,1 / 2] .
$$

We now compute the left hand side of inequality (5.2) for

$$
g:=f \circ g_{a} .
$$


Let $p<\infty$. Then we have, for all $t \in[-1 / 2,1 / 2]$,

$$
\begin{aligned}
& \int_{Q_{0}}\left|\frac{\partial\left(f \circ g_{a}\right)}{\partial \nu}(x+t \nu)-\frac{\partial\left(f \circ g_{a}\right)}{\partial \nu}(x)\right|^{p} d x= \\
& =\int_{-1 / 2}^{1 / 2}\left|a f^{\prime}\left(a\left(x_{1}+t\right)\right)-a f^{\prime}\left(a x_{1}\right)\right|^{p} d x_{1}=a^{p-1} \int_{-a / 2}^{a / 2}\left|f^{\prime}(y+a t)-f^{\prime}(y)\right|^{p} d y .
\end{aligned}
$$

Then by setting $g:=f \circ g_{a}$ in (5.2), and by exploiting inequality (1.3), and by setting $w=a t$, and by inequality $a>1$, we conclude that

$$
\begin{aligned}
a^{p-1} h(w / a)^{-p} \int_{-a / 2}^{a / 2}\left|f^{\prime}(y+w)-f^{\prime}(y)\right|^{p} d y & \leq A^{p}\left\|f \circ g_{a}\right\|_{E}^{p} \\
& \leq(A B)^{p} a^{p}(N(\varphi)+1)^{p}
\end{aligned}
$$

for all $w \in]-a / 2, a / 2\left[\backslash\{0\}\right.$. By dividing both hand sides by $a^{p}$, and by taking the limit as $a$ tends to $+\infty$, we obtain

$\limsup _{a \rightarrow+\infty} a^{-1} h(w / a)^{-p} \int_{-a / 2}^{a / 2}\left|f^{\prime}(y+w)-f^{\prime}(y)\right|^{p} d y \leq(A B)^{p}(N(\varphi)+1)^{p}<\infty$.

By (5.1), we have

$$
\lim _{a \rightarrow+\infty} a^{-1} h(w / a)^{-p}=\frac{1}{|w|} \lim _{y \rightarrow 0}|y| h(y)^{-p}=+\infty .
$$

Hence,

$$
\int_{-\infty}^{+\infty}\left|f^{\prime}(y+w)-f^{\prime}(y)\right|^{p} d y=0 \quad \forall w \in \mathbb{R} \backslash\{0\} .
$$

Hence, $f^{\prime}(y+w)-f^{\prime}(y)=0$ a.e. By the continuity of $f^{\prime}$, and by the arbitrariness of $w$, we conclude that $f^{\prime}$ must be a constant. The case $p=\infty$ follows by similar arguments.

For $p=1$, we have the following variant.

Proposition 12 Let $N$ be a norm on $\mathcal{D}\left(\mathbb{R}^{n}\right)$. Let $h: \mathbb{R} \rightarrow[0,+\infty[$ be a function such that $h(t)>0$ if $t \in \mathbb{R} \backslash\{0\}$, and such that $\lim _{t \rightarrow 0} h(t)=0$. Let $E$ be a subspace of $W_{1, \text { loc }}^{2}\left(\mathbb{R}^{n}\right)$ containing $\mathcal{D}\left(\mathbb{R}^{n}\right)$ such that there exist a direction $\nu \in \mathbb{R}^{n},|\nu|=1$, and a positive constant $A$ such that

$$
\int_{Q}\left|\frac{\partial^{2} g}{\partial \nu^{2}}(x+t \nu)-\frac{\partial^{2} g}{\partial \nu^{2}}(x)\right| d x \leq A h(t)\|g\|_{E},
$$

for all $g \in E$, for all cubes $Q \subset \mathbb{R}^{n}$ with $|Q|=1$, and for all $t \in[-1 / 2,1 / 2]$.

If there exist a twice continuously differentiable function $f: \mathbb{R} \rightarrow \mathbb{R}$ and a constant $B>0$ such that $T_{f}$ maps $\mathcal{D}\left(\mathbb{R}^{n}\right)$ into $E$, and such that the inequality (1.3) holds, then $f$ must be an affine function. 
Proof. Arguing as in the previous proof, and exploiting the continuity of $f^{\prime \prime}$, we obtain that

$$
f^{\prime \prime}(y+w)-f^{\prime \prime}(y)=0,
$$

for all $y, w \in \mathbb{R}$. Hence, there exist $\alpha, \beta, \gamma \in \mathbb{R}$ such that $f(x)=\alpha x^{2}+\beta x+\gamma$ for all $x \in \mathbb{R}$. By inequality (1.3), and by taking $g:=g_{a}$ as in (5.3), we obtain

$$
\left\|\alpha a^{2} \varphi^{2}+\beta a \varphi+\gamma\right\|_{E} \leq B(N(a \varphi)+1) \quad \forall a>1 .
$$

Then by taking the limit as $a$ tends to infinity, we obtain $\alpha=0$.

Remark. Besov spaces with $s>1+(1 / p)$ satisfy the assumptions of Proposition 11 if $p>1$ and of Proposition 12 if $p=1$. Thus triviality of sublinear superposition operators holds in these Besov spaces. However, Propositions 11 and 12 may be used also in the context of a refined scale of function spaces. Namely, the so-called Besov spaces of generalized smoothness (cf. Gol'dman [17] and Farkas and Leopold [13]).

\subsection{The main theorem on the S.O.P. for Besov spaces}

Bourdaud and Kateb [8] have proved the following result.

Theorem 6 Let $p \in] 1,+\infty[, q \in[1,+\infty], 0<s<1+(1 / p)$. If the function $f$ belongs to $U_{p}^{1}(\mathbb{R})$ and $f(0)=0$, then $T_{f}\left(B_{p, q}^{s}\left(\mathbb{R}^{n}\right)\right) \subseteq B_{p, q}^{s}\left(\mathbb{R}^{n}\right)$.

The proof of Bourdaud and Kateb relies on a delicate argument of first order spline approximation. We now prove a result for $T_{f}$ in the critical case $s=1+1 / p$. Namely, that $T_{f}$ maps $B V_{p}^{1}(\mathbb{R})$ to $\dot{B}_{p, \infty}^{1+(1 / p)}(\mathbb{R})$, and then we shall derive Theorem 6 by nonlinear interpolation. Thus we now prove the following.

Theorem 7 Let $p \in] 1,+\infty\left[\right.$. If $f \in U_{p}^{1}(\mathbb{R})$ and $g \in B V_{p}^{1}(\mathbb{R})$, then $f \circ g$ belongs to $\dot{B}_{p, \infty}^{1+(1 / p)}(\mathbb{R})$. Moreover, there exists $c_{p}>0$ such that

$$
\|f \circ g\|_{\dot{B}_{p, \infty}^{1+(1 / p)}(\mathbb{R})} \leq c_{p}\|f\|_{U_{p}^{1}(\mathbb{R})}\left\|g^{\prime}\right\|_{B V_{p}(\mathbb{R})} \quad \forall g \in B V_{p}^{1}(\mathbb{R}) .
$$

Proof. We first prove inequality (5.5) in case $g$ is a real analytic function. Thus we introduce the following.

Lemma 2 Let $p \in\left[1,+\infty\left[\right.\right.$. If $g$ is a real analytic function in $B V_{p}^{1}(\mathbb{R})$, and if $\Phi \in \mathcal{U}_{p}(g(\mathbb{R}))$ is a Borel measurable function bounded on $g(\mathbb{R})$, then

$$
\begin{aligned}
& \int_{\mathbb{R}}|\Phi(g(x+h))-\Phi(g(x))|^{p}\left|g^{\prime}(x)\right|^{p} d x \\
& \quad \leq \max ^{p}\left\{\left\|g^{\prime}\right\|_{\infty}, \nu_{p}\left(g^{\prime}\right)\right\}\left(2^{p} \sup _{g(\mathbb{R})}|\Phi|^{p}+\|\Phi\|_{\mathcal{U}_{p}(g(\mathbb{R}))}^{p}\right)|h| \quad \forall h \in \mathbb{R} .
\end{aligned}
$$


Proof of Lemma 2. Let $g$ be a real analytic function in $B V_{p}^{1}$ not identically equal to zero. Then the set of zeros of $g^{\prime}$ is discrete, and its complement in $\mathbb{R}$ is the union of a finite or countable family $\left\{I_{l}\right\}_{l \in \mathbb{L}}$ of nonempty open disjoint intervals of $\mathbb{R}$. Clearly, $g_{l}:=g_{\mid I_{l}}$ is a diffeomorphism of $I_{l}$ onto $g\left(I_{l}\right)$. Now let $h \in \mathbb{R}$. We consider for example the case $h>0$. We set

$$
I_{l}^{\prime}:=I_{l} \backslash\left[\sup I_{l}-h, \sup I_{l}\right], \quad I_{l}^{\prime \prime}:=I_{l} \backslash I_{l}^{\prime},
$$

with the understanding that if $\sup I_{l}=+\infty$, then $I_{l}^{\prime}:=I_{l}$. We note that $I_{l}^{\prime}$ as well as $I_{l}^{\prime \prime}$ can be empty. We set $J_{l}^{\prime}:=g_{l}\left(I_{l}^{\prime}\right)$. If $I_{l}^{\prime} \neq \emptyset$, then we have

$$
\left.\left|g\left(g_{l}^{-1}(y)+h\right)-y\right| \leq \sup _{I_{l}}\left|g^{\prime}\right|\right)|h| \quad \text { for } \quad y \in J_{l}^{\prime},
$$

where $g_{l}^{-1}$ denotes the inverse function of $g_{l}$. Hence,

$$
\begin{aligned}
\int_{I_{l}^{\prime}} \mid \Phi & (g(x+h))-\left.\Phi(g(x))\right|^{p}\left|g^{\prime}(x)\right|^{p} d x \leq \\
& \leq \sup _{I_{l}}\left|g^{\prime}\right|^{p-1} \int_{J_{l}^{\prime}}\left|\Phi\left(y+\left(g\left(g_{l}^{-1}(y)+h\right)-y\right)\right)-\Phi(y)\right|^{p} d y \\
& \leq\|\Phi\|_{\mathcal{U}_{p}(g(\mathbb{R}))}^{p}\left(\sup _{I_{l}}\left|g^{\prime}\right|^{p}\right)|h| .
\end{aligned}
$$

Also, we note that such inequality holds trivially in case $I_{l}^{\prime}=\emptyset$. Since the length of $I_{l}^{\prime \prime}$ is at most $|h|$, we have

$$
\int_{I_{l}^{\prime \prime}}|\Phi(g(x+h))-\Phi(g(x))|^{p}\left|g^{\prime}(x)\right|^{p} d x \leq 2^{p}\left(\sup _{g(\mathbb{R})}|\Phi|^{p}\right)\left(\sup _{I_{l}^{\prime \prime}}\left|g^{\prime}\right|^{p}\right)|h| .
$$

Then we conclude that

$$
\begin{aligned}
\int_{I_{l}} \mid \Phi(g(x+h))- & \left.\Phi(g(x))\right|^{p}\left|g^{\prime}(x)\right|^{p} d x \\
\leq & \left(\sup _{I_{l}}\left|g^{\prime}\right|^{p}\right)\left(2^{p} \sup _{g(\mathbb{R})}|\Phi|^{p}+\|\Phi\|_{\mathcal{U}_{p}(g(\mathbb{R}))}^{p}\right)|h| .
\end{aligned}
$$

If $\mathbb{L}$ contains only one element, then $I_{l}=\mathbb{R}$, and inequality (5.6) follows from (5.8). Then we can assume that $\mathbb{L}$ has at least two elements. Now we note that by construction of the intervals $I_{l}$, the derivative of $g$ vanishes at the endpoints of $I_{l}$ in $\mathbb{R}$. We denote by $\beta_{l}$ one of such endpoints. Now let $\widetilde{\mathbb{L}}:=\left\{l \in \mathbb{L}: I_{l}\right.$ is bounded $\}$. Clearly, $\mathbb{L} \backslash \widetilde{\mathbb{L}}$ has at most two elements. If $l \in \widetilde{\mathbb{L}}$ then there exists $\xi_{l} \in I_{l}$ such that

$$
\left|g^{\prime}\left(\xi_{l}\right)\right|=\sup _{I_{l}}\left|g^{\prime}\right|
$$


Now let $l \in \mathbb{L} \backslash \widetilde{\mathbb{L}}, \epsilon>0$. Then there exists $\xi_{l} \in I_{l}$ such that

$$
\left|g^{\prime}\left(\xi_{l}\right)\right|^{p} \geq \sup _{I_{l}}\left|g^{\prime}\right|^{p}-\epsilon .
$$

The open intervals with endpoints $\xi_{l}$ and $\beta_{l}(l \in \mathbb{L})$ are clearly pairwise disjoint. Then we have

$\sum_{l \in \mathbb{L}} \sup _{I_{l}}\left|g^{\prime}\right|^{p} \leq \sum_{l \in \widetilde{\mathbb{L}}}\left|g^{\prime}\left(\xi_{l}\right)-g^{\prime}\left(\beta_{l}\right)\right|^{p}+\sum_{l \in \mathbb{L} \backslash \widetilde{\mathbb{L}}}\left|g^{\prime}\left(\xi_{l}\right)-g^{\prime}\left(\beta_{l}\right)\right|^{p}+2 \epsilon \leq \nu_{p}^{p}\left(g^{\prime}\right)+2 \epsilon$.

By arbitrariness of $\epsilon$, we have

$$
\sum_{l \in \mathbb{L}} \sup _{I_{l}}\left|g^{\prime}\right|^{p} \leq \nu_{p}^{p}\left(g^{\prime}\right)
$$

By inequality (5.8), we conclude that inequality (5.6) holds.

Now we go back to the proof of Theorem 7 . Let $\Phi$ be a bounded Borel measurable representative of $f^{\prime}$ of class $\mathcal{U}_{p}(\mathbb{R})$. Assume that $g^{\prime}$ is normalized. Then the equality

$$
(f \circ g)^{\prime}=(\Phi \circ g) g^{\prime}
$$

for the distributional derivatives holds for all $g \in B V_{p}^{1}(\mathbb{R})$. We first prove inequality (5.5) for a real analytic function $g \in B V_{p}^{1}(\mathbb{R})$. By Lemma 2 and by Theorem 5 , there exists $c_{p}>0$ such that

$$
\begin{aligned}
\left(\int_{\mathbb{R}} \mid \Phi(g(x+\right. & \left.h)) g^{\prime}(x+h)-\left.\Phi(g(x)) g^{\prime}(x)\right|^{p} d x\right)^{1 / p} \\
\leq & \left(\int_{\mathbb{R}}|\Phi(g(x+h))|^{p}\left|g^{\prime}(x+h)-g^{\prime}(x)\right|^{p} d x\right)^{1 / p} \\
& +\left(\int_{\mathbb{R}}|\Phi(g(x+h))-\Phi(g(x))|^{p}\left|g^{\prime}(x)\right|^{p} d x\right)^{1 / p} \\
\leq & c_{p}\left(\sup _{\mathbb{R}}|\Phi|+\|\Phi\|_{\mathcal{U}_{p}(\mathbb{R})}\right)\left\|g^{\prime}\right\|_{B V_{p}(\mathbb{R})}|h|^{1 / p},
\end{aligned}
$$

holds for all $h \in \mathbb{R}$, and for all real analytic functions $g$ in $B V_{p}^{1}(\mathbb{R})$. Then we conclude that inequality (5.5) holds for $g \in B V_{p}^{1}(\mathbb{R})$ real analytic.

We now prove the same inequality in the general case in which $g \in$ $B V_{p}^{1}(\mathbb{R})$. To do so, we use a sequence $\left(\phi_{j}\right)_{j>0}$ of real analytic mollifiers defined by

$$
\phi_{j}(x):=j \phi(j x) \quad \forall x \in \mathbb{R},
$$

where the Fourier transform $\hat{\phi}$ belongs to $\mathcal{D}(\mathbb{R})$ and $\hat{\phi}(0)=1$. 
Then we set $g_{j}:=g * \phi_{j}$. By translation invariance of $B V_{p}(\mathbb{R})$, we have

$$
\left\|g_{j}^{\prime}\right\|_{B V_{p}(\mathbb{R})} \leq\left\|g^{\prime}\right\|_{B V_{p}(\mathbb{R})} .
$$

Applying inequality (5.5) to $g_{j}$ yields

$$
\left\|f \circ g_{j}\right\|_{\dot{B}_{p, \infty}^{1+(1 / p)}(\mathbb{R})} \leq c_{p}\|f\|_{U_{p}^{1}(\mathbb{R})}\left\|g^{\prime}\right\|_{B V_{p}(\mathbb{R})} .
$$

Since $g$ is Lipschitz continuous, we have $\lim _{j \rightarrow \infty} g_{j}(x)=g(x)$ for all $x \in \mathbb{R}$. Hence, the continuity of $f$ implies that

$$
\lim _{j \rightarrow \infty} f\left(g_{j}(x)\right)=f(g(x)),
$$

for all $x \in \mathbb{R}$. Moreover,

$$
\left|f\left(g_{j}(x)\right)\right| \leq|f(0)|+\left(\sup _{\mathbb{R}}|\Phi|\right)\left(\sup _{j}\left|g_{j}(0)\right|+|x|\left\|g_{j}^{\prime}\right\|_{\infty}\right) .
$$

Since $\left\|g_{j}^{\prime}\right\|_{\infty} \leq\left\|g_{j}^{\prime}\right\|_{B V_{p}(\mathbb{R})}$, inequality (5.11) implies that $\sup _{j \in \mathbb{N}}\left\|g_{j}^{\prime}\right\|_{\infty}<$ $+\infty$. Thus we conclude that $\lim _{j \rightarrow \infty} f \circ g_{j}=f \circ g$ in the sense of distributions. By exploiting the Fatou property of $\dot{B}_{p, \infty}^{1+(1 / p)}(\mathbb{R})$ (see Subsection 7.2), we deduce that $f \circ g \in \dot{B}_{p, \infty}^{1+(1 / p)}(\mathbb{R})$, and that inequality (5.5) holds.

\subsection{Superposition operators in Besov spaces}

We are now in the position to derive some consequences of Theorem 7 . By combining Theorem 5, Proposition 10 and Theorem 7, we immediately deduce the validity of the following.

Theorem 8 Let $p \in] 1,+\infty\left[\right.$. If $f \in U_{p}^{1}(\mathbb{R})$ and $g \in \dot{B}_{p, 1}^{1+(1 / p)}(\mathbb{R})$, then $f \circ g \in \dot{B}_{p, \infty}^{1+(1 / p)}(\mathbb{R})$. Moreover, there exists a constant $c_{p}>0$ such that

$$
\|f \circ g\|_{\dot{B}_{p, \infty}^{1+(1 / p)}(\mathbb{R})} \leq c_{p}\|f\|_{U_{p}^{1}(\mathbb{R})}\left(\|g\|_{\dot{B}_{p, 1}^{1+(1 / p)}(\mathbb{R})}+\left|L_{g^{\prime}}\right|\right) \quad \forall g \in \dot{B}_{p, 1}^{1+(1 / p)}(\mathbb{R}) .
$$

Then for nonhomogeneous Besov spaces, we have the following.

Theorem 9 Let $p \in] 1,+\infty\left[\right.$. Let $f \in U_{p}^{1}(\mathbb{R})$ be such that $f(0)=0$. If $g \in B_{p, 1}^{1+(1 / p)}(\mathbb{R})$, then $f \circ g$ belongs to $B_{p, \infty}^{1+(1 / p)}(\mathbb{R})$. Moreover, there exists $c_{p}>0$ such that

$$
\|f \circ g\|_{B_{p, \infty}^{1+(1 / p)}(\mathbb{R})} \leq c_{p}\|f\|_{U_{p}^{1}(\mathbb{R})}\|g\|_{B_{p, 1}^{1+(1 / p)}(\mathbb{R})} \quad \forall g \in B_{p, 1}^{1+(1 / p)}(\mathbb{R}) .
$$


Proof. Since $f(0)=0$, we have $|f(t)| \leq\left\|f^{\prime}\right\|_{\infty}|t|$, for all $t \in \mathbb{R}$. Hence,

$$
\|f \circ g\|_{p} \leq\left\|f^{\prime}\right\|_{\infty}\|g\|_{p} \quad \forall g \in B_{p, 1}^{1+(1 / p)}(\mathbb{R}) .
$$

Now we note that the membership of $g$ in $B_{p, 1}^{1+(1 / p)}(\mathbb{R})$ implies that

$$
g^{\prime} \in L^{p}(\mathbb{R}) \cap \dot{B}_{p, 1}^{1 / p}(\mathbb{R}) .
$$

Hence, we must have $L_{g^{\prime}}=0$. Then we can conclude by combining Theorem 8 , with inequality $(5.14)$.

By nonlinear interpolation we now obtain a new proof of Theorem 6 in case $n=1$.

Theorem 10 Let $p \in] 1,+\infty[, s \in] 0,1+(1 / p)\left[, q \in[1,+\infty]\right.$. Let $f \in U_{p}^{1}(\mathbb{R})$ be such that $f(0)=0$. If $g \in B_{p, q}^{s}(\mathbb{R})$, then $f \circ g \in B_{p, q}^{s}(\mathbb{R})$. Moreover, there exists $c>0$ such that

$$
\|f \circ g\|_{B_{p, q}^{s}(\mathbb{R})} \leq c\|f\|_{U_{p}^{1}(\mathbb{R})}\|g\|_{B_{p, q}^{s}(\mathbb{R})} \quad \forall g \in B_{p, q}^{s}(\mathbb{R}) .
$$

Proof. We plan to apply the classical nonlinear interpolation theorem of Peetre [23], which we now introduce. Let $1 \leq q \leq \infty$ and $0<\theta<1$. Let $\left(A_{0}, A_{1}\right)$ and $\left(B_{0}, B_{1}\right)$ be compatible couples of Banach spaces such that $A_{1} \hookrightarrow A_{0}$ and $B_{1} \hookrightarrow B_{0}$. Let $T$ be a map from $A_{0}$ to $B_{0}$ such that $T(0)=0$, and such that there exists $c>0$ with

$$
\left\|T g_{1}-T g_{2}\right\|_{B_{0}} \leq c\left\|g_{1}-g_{2}\right\|_{A_{0}} \forall g_{1}, g_{2} \in A_{0},
$$

and with

$$
\|T g\|_{B_{1}} \leq c\|g\|_{A_{1}} \quad \forall g \in A_{1} .
$$

Then by Peetre's Theorem, $T$ maps $\left(A_{0}, A_{1}\right)_{\theta, q}$ into $\left(B_{0}, B_{1}\right)_{\theta, q}$ and there exists $c^{\prime}>0$ such that

$$
\|T g\|_{\left(B_{0}, B_{1}\right)_{\theta, q}} \leq c^{\prime}\|g\|_{\left(A_{0}, A_{1}\right)_{\theta, q}} \quad \forall g \in\left(A_{0}, A_{1}\right)_{\theta, q} .
$$

Now we choose

$$
A_{0}=B_{0}:=L^{p}\left(\mathbb{R}^{n}\right), A_{1}:=B_{p, 1}^{1+(1 / p)}(\mathbb{R}), B_{1}:=B_{p, \infty}^{1+(1 / p)}(\mathbb{R})
$$

and

$$
\theta:=\frac{s}{1+(1 / p)} .
$$

Thus Theorem 10 follows by Theorem 9 and by classical interpolation properties of Besov spaces, (cf. e.g., Bergh and Löfstrom [3, 6.4.5], Peetre [24], Triebel $[30,2.4 .2]$.

Remark. Theorem 10 has a counterpart for homogeneous Besov spaces, see $[10]$. 


\section{An extension to the $n$-dimensional case}

We do not know how to define $B V_{p}^{1}\left(\mathbb{R}^{n}\right)$ in order to generalize Theorems 4 and 7. Nevertheless, we can show that Theorems 9 and 10 can be extended to Besov spaces in $\mathbb{R}^{n}$. We do so by means of the following.

Theorem 11 Let $p \in] 1,+\infty[, q \in[1,+\infty]$, and $0<s<1+(1 / p)$. If $f \in U_{p}^{1}(\mathbb{R})$ and $f(0)=0$, then $T_{f}$ maps

(i) $B_{p, 1}^{1+(1 / p)}\left(\mathbb{R}^{n}\right)$ into $B_{p, \infty}^{1+(1 / p)}\left(\mathbb{R}^{n}\right)$,

(ii) $B_{p, q}^{s}\left(\mathbb{R}^{n}\right)$ into $B_{p, q}^{s}\left(\mathbb{R}^{n}\right)$.

Moreover, there exists a constant $c>0$ such that

$$
\|f \circ g\| \leq c\|f\|_{U_{p}^{1}(\mathbb{R})}\|g\|
$$

holds for all $g$ in the various function spaces in (i), (ii), with their respective norms.

Proof. As it is well known, a tempered distribution $g$ of $L_{\ell o c}^{p}\left(\mathbb{R}^{n}\right)$ belongs to $B_{p, q}^{s}\left(\mathbb{R}^{n}\right)$ (with $0<s<2,1 \leq p<+\infty, 1 \leq q<+\infty$ ) if and only if

$$
\|g\|_{p}+\sum_{j=1}^{n}\left(\int_{\mathbb{R}}\left(\frac{1}{|h|^{s}}\left(\int_{\mathbb{R}^{n}}\left|g\left(x+h e_{j}\right)+g\left(x-h e_{j}\right)-2 g(x)\right|^{p} d x\right)^{1 / p}\right)^{q} \frac{d h}{|h|}\right)^{1 / q}
$$

$$
<+\infty,
$$

where $e_{1}, \ldots, e_{n}$ denotes the canonical basis in $\mathbb{R}^{n}$, and the above expression is an equivalent norm in $B_{p, q}^{s}\left(\mathbb{R}^{n}\right)$ (cf. e.g., Triebel [31, thm. 2.6.1]), and a corresponding statement holds for $q=+\infty$ or $p=+\infty$ with obvious modifications.

We divide our proof into two steps.

Step 1. We first prove statement (i). For the sake of simplicity, we consider only case $n=2$. Let

$$
g \in B_{p, 1}^{1+(1 / p)}\left(\mathbb{R}^{2}\right) \quad \text { and } \quad g_{x}(y):=g(x, y) .
$$

By the Minkowski inequality, we have

$$
\begin{aligned}
& \left(\int_{\mathbb{R}}\left(\int_{\mathbb{R}}\left(\int_{\mathbb{R}}|g(x, y+h)+g(x, y-h)-2 g(x, y)|^{p} d y\right)^{1 / p}|h|^{-2-(1 / p)} d h\right)^{p} d x\right)^{1 / p} \\
& \leq \int_{\mathbb{R}}\left(\iint_{\mathbb{R}^{2}}|g(x, y+h)+g(x, y-h)-2 g(x, y)|^{p} d x d y\right)^{1 / p}|h|^{-2-(1 / p)} d h .
\end{aligned}
$$


Using the alternative norm (6.1) with $q=1$, we deduce that

$$
g_{x} \in B_{p, 1}^{1+(1 / p)}(\mathbb{R})
$$

for almost every $x \in \mathbb{R}$, and that there exists $c>0$ such that

$$
\left(\int_{\mathbb{R}}\left\|g_{x}\right\|_{B_{p, 1}^{1+(1 / p)}(\mathbb{R})}^{p} d x\right)^{1 / p} \leq c\|g\|_{B_{p, 1}^{1+(1 / p)}\left(\mathbb{R}^{2}\right)} \quad \forall g \in B_{p, 1}^{1+(1 / p)}\left(\mathbb{R}^{2}\right) .
$$

Now we have

$$
\begin{aligned}
& \sup _{h \neq 0}|h|^{-1-(1 / p)}\left(\iint_{\mathbb{R}^{2}}|f(g(x, y+h))+f(g(x, y-h))-2 f(g(x, y))|^{p} d x d y\right)^{1 / p} \\
& \leq\left(\int_{\mathbb{R}}\left(\sup _{h \neq 0}|h|^{-p-1} \int_{\mathbb{R}}|f(g(x, y+h))+f(g(x, y-h))-2 f(g(x, y))|^{p} d y\right) d x\right)^{1 / p} \\
& \leq c\left(\int_{\mathbb{R}}\left\|f \circ g_{x}\right\|_{B_{p, \infty}^{1+(1 / p)}(\mathbb{R})}^{p} d x\right)^{1 / p} \quad \forall g \in B_{p, 1}^{1+(1 / p)}\left(\mathbb{R}^{2}\right) .
\end{aligned}
$$

By inequality (6.2) and by Theorem 9, we deduce that

$$
\begin{aligned}
& \sup _{h \neq 0}|h|^{-1-(1 / p)}\left(\iint_{\mathbb{R}^{2}}|f(g(x, y+h))+f(g(x, y-h))-2 f(g(x, y))|^{p} d x d y\right)^{1 / p} \\
& \leq c\|f\|_{U_{p}^{1}(\mathbb{R})}\|g\|_{B_{p, 1}^{1+(1 / p)}\left(\mathbb{R}^{2}\right)} \quad \forall g \in B_{p, 1}^{1+(1 / p)}\left(\mathbb{R}^{2}\right) .
\end{aligned}
$$

Then interchanging the role of variables and using the alternative norm (6.1), with $q=\infty$, we obtain

$$
\|f \circ g\|_{B_{p, \infty}^{1+(1 / p)}\left(\mathbb{R}^{2}\right)} \leq c\|f\|_{U_{p}^{1}(\mathbb{R})}\|g\|_{B_{p, 1}^{1+(1 / p)}\left(\mathbb{R}^{2}\right)} .
$$

Step 2. Property (ii) follows by property (i) and by nonlinear interpolation, as in Theorem 10.

Remark. Theorem 11, part (ii), is precisely Theorem 6, and it has now been deduced by nonlinear interpolation, and by the corresponding result for the critical value $s=1+(1 / p)$. In a later work, Kateb [19] has improved Theorem 6 by showing that the condition $f \in U_{p}^{1}$ can be replaced by condition $f^{\prime} \in \dot{B}_{p, \infty}^{1 / p} \cap L^{\infty}$. We do not know if such weaker condition still implies that $T_{f}$ maps $B_{p, 1}^{1+(1 / p)}\left(\mathbb{R}^{n}\right)$ into $B_{p, \infty}^{1+(1 / p)}\left(\mathbb{R}^{n}\right)$. In particular, we do not know if our method could be applied to obtain a simpler proof of the last theorem of Kateb. 


\section{Appendix}

7.1. Diversity of the classes $B V_{p}, U_{p}, \dot{B}_{p, \infty}^{1 / p}$

Proposition 13 Let $p \in] 1,+\infty[$. The two inclusions

$$
B V_{p}(\mathbb{R}) \subset U_{p}(\mathbb{R}) \cap L^{\infty}(\mathbb{R}) \subset \dot{B}_{p, \infty}^{1 / p}(\mathbb{R}) \cap L^{\infty}(\mathbb{R}) .
$$

are proper.

Proof. Step 1. We shall employ ideas of Bourdaud [7]. We first introduce the function

$$
f(x):=x \rho(x) \sin |x|^{-p} \quad \forall x \in \mathbb{R} \backslash\{0\}, f(0):=0,
$$

where $\rho \in \mathcal{D}(\mathbb{R})$ and

$$
\rho(x)=1 \quad \text { for }|x| \leq 1, \quad \rho(x)=0 \quad \text { for }|x| \geq 2, \quad 0 \leq \rho \leq 1 .
$$

We now prove that $f \in \mathcal{U}_{p}(\mathbb{R})$. Indeed, we just need to estimate

$$
I(t):=\int_{-2}^{2}|x|^{p} \sup _{|h| \leq t}|\sin | x+\left.h\right|^{-p}-\left.\sin |x|^{-p}\right|^{p} d x
$$

for $|t| \leq 1 / 4$. Clearly,

$$
|| x+\left.h\right|^{-p}-\left.|x|^{-p}\left|\leq p 2^{p+1}\right| h|| x\right|^{-p-1} \quad \text { for } \quad 0<|h| \leq|x| / 2,
$$

and

$$
2 t \leq t^{1 /(p+1)} .
$$

Hence, there exists $c_{p}>0$ such that

$$
\begin{aligned}
I(t) & \leq p^{p} 2^{p(p+1)}\left(t^{p} \int_{t^{1 /(p+1)} \leq|x| \leq 2}|x|^{p}|x|^{-p(p+1)} d x+\int_{|x|<t^{1 /(p+1)}}|x|^{p} d x\right) \\
& \leq c_{p} t
\end{aligned}
$$

for all $t \in[-1 / 4,1 / 4]$, an inequality which shows that $f \in \mathcal{U}_{p}(\mathbb{R})$.

We now prove that $f \notin \mathcal{V}_{p}(\mathbb{R})$. To do so, we set

$$
\begin{aligned}
a_{k} & :=(k \pi+(\pi / 2))^{-1 / p}, \\
b_{k} & :=(k \pi)^{-1 / p},
\end{aligned}
$$

for any integer $k \geq 1$. 
The intervals $\left[a_{k}, b_{k}\right]$ are pairwise disjoint and

$$
\sum_{k=1}^{N}\left|f\left(b_{k}\right)-f\left(a_{k}\right)\right|^{p}=\sum_{k=1}^{N} a_{k}^{p}=\sum_{k=1}^{N} \frac{1}{(k+(1 / 2)) \pi},
$$

for all $N \geq 1$. Hence, the divergent character of the sum in the right hand side for $N$ tending to infinity implies that $f \notin \mathcal{V}_{p}(\mathbb{R})$. The continuity of $f$ guarantees that $f$ cannot be a representative of an element of $B V_{p}(\mathbb{R})$.

Step 2. We now prove that the second inclusion of the statement is proper. Let $\varphi \in \mathcal{D}(\mathbb{R})$ be an even function decreasing on $[0,+\infty[$. Let the support of $\varphi$ be contained in $[-2,2]$. Let $0 \leq \varphi \leq 1$ and $\varphi(0)=1$. Then we introduce the functions

$$
f_{n}(x):=\frac{1}{n} \sum_{j=1}^{n} \varphi\left(2^{j} x\right) \quad \text { and } \quad g_{n}(x):=\sum_{k=1}^{n} f_{n}(x-3 k) \quad(n=1,2, \ldots) .
$$

Clearly, $f_{n}$ is a $C^{\infty}$ function with support contained in $[-1,1]$, such that $0 \leq f_{n} \leq 1$ and $f_{n}(0)=1$. Since the intervals [3k-1,3k+1] are pairwise disjoint, we have $0 \leq g_{n} \leq 1$. Moreover, the support of $g_{n}$ is contained in $[2,3 n+1]$.

We now define inductively a sequence of integers $\left(l_{k}\right)_{k \geq 1}$ as follows

$$
l_{1}:=0, \quad l_{n+1}:=3 n+l_{n} \quad(n=1,2, \ldots) .
$$

Then we define the sequence of functions

$$
G_{n}(x):=g_{n}\left(x-l_{n}\right) \quad(n=1,2, \ldots) .
$$

Clearly, $\operatorname{dist}\left(\operatorname{supp} G_{n}, \operatorname{supp} G_{m}\right) \geq 1$ for $n \neq m$. Hence, the function

$$
G(x):=\sum_{n=1}^{\infty} \alpha_{n} G_{n}(x)
$$

is defined and continuous, for every sequence $\left(\alpha_{n}\right)_{n \geq 1}$ of real numbers. Clearly, $0 \leq G \leq 1$ if $0 \leq \alpha_{n} \leq 1$ for all $n$.

If $M^{-1}:=\max \left\{\|\varphi\|_{\infty}, 4\left\|\varphi^{\prime}\right\|_{\infty}\right\}$, then the elementary building blocks

$$
M \varphi\left(2^{j} x-2^{j} l_{n}-3 k 2^{j}\right)
$$

are $(1 / p, p)$-atoms in the sense of Frazier and Jawerth. 
By Frazier and Jawerth [16, p. 785$]$, there exists $c>0$ such that

$$
\begin{aligned}
\|G\|_{\dot{B}_{p, \infty}^{1 / p}(\mathbb{R})} & \leq c \sup _{j \geq 1}\left(\sum_{n=1}^{\infty} \sum_{k=1}^{n} \alpha_{n}^{p} n^{-p}\right)^{1 / p}=c\left(\sum_{n=1}^{\infty} \sum_{k=1}^{n} \alpha_{n}^{p} n^{-p}\right)^{1 / p} \\
& =c\left(\sum_{n=1}^{\infty} \alpha_{n}^{p} n^{1-p}\right)^{1 / p} .
\end{aligned}
$$

Finally, we observe that

$$
\int_{\mathbb{R}} \sup _{0 \leq h \leq 1}|G(x+h)-G(x)|^{p} d x \geq \sum_{n=1}^{\infty}\left|\alpha_{n}\right|^{p} \int_{\mathbb{R}} \sup _{0 \leq h \leq 1}\left|g_{n}(x+h)-g_{n}(x)\right|^{p} d x .
$$

Now we note that inequalities $3 k-1 \leq x \leq 3 k$ and $0 \leq h \leq 1$ imply $3 k-1 \leq x+h \leq 3 k+1$, and thus

$$
g_{n}(x+h)=f_{n}(x+h-3 k) .
$$

Hence,

$$
\|G\|_{\mathcal{U}_{p}(\mathbb{R})}^{p} \geq \sum_{n=1}^{\infty}\left|\alpha_{n}\right|^{p} \sum_{k=1}^{n} \int_{-1}^{0} \sup _{0 \leq h \leq 1}\left|f_{n}(x+h)-f_{n}(x)\right|^{p} d x .
$$

Taking $h:=-x$, we see that

$$
\sup _{0 \leq h \leq 1}\left|f_{n}(x+h)-f_{n}(x)\right|^{p} \geq\left|f_{n}(0)-f_{n}(x)\right|^{p}
$$

for all $x \in[-1,0]$. Since

$$
f_{n}(1 / 2)=\frac{\varphi(1)}{n} \leq 1 / n \quad \text { for } n \geq 2,
$$

we have

$$
\left|f_{n}(0)-f_{n}(x)\right| \geq 1 / 2 \quad \text { for } x \in[-1,-1 / 2] \text { and } n \geq 2 .
$$

Hence,

$$
\|G\|_{\mathcal{U}_{p}(\mathbb{R})}^{p} \geq 2^{-p} \sum_{n=2}^{\infty} n\left|\alpha_{n}\right|^{p}
$$

Taking $\alpha_{n}:=n^{-2 / p}$, we obtain $G \notin \mathcal{U}_{p}(\mathbb{R})$. The above argument also shows that if a measurable function equals $G$ almost everywhere, then it cannot belong to $\mathcal{U}_{p}(\mathbb{R})$. Hence, $G$ cannot define an element of $U_{p}(\mathbb{R})$, although $G \in \dot{B}_{p, \infty}^{1 / p}(\mathbb{R})$. 
Remark. We now show that there exist simple examples of unbounded functions in $\dot{B}_{p, \infty}^{1 / p}(\mathbb{R}) \backslash U_{p}(\mathbb{R})$. Let $\rho$ be as in the previous proof. Then we set

$$
f(x):=|\log | x|| \rho(x) \quad \forall x \in \mathbb{R} \backslash\{0\}, \quad f(0):=0,
$$

By [7, prop. 2], we have $f \in \dot{B}_{p, \infty}^{1 / p}(\mathbb{R})$. Instead

$$
\sup _{|h| \leq t}|f(x+h)-f(x)|=+\infty
$$

for all $x \in] 0, t]$, and all $t>0$. Hence, $f$ cannot define an element of $U_{p}(\mathbb{R})$. We do not know of simple explicit examples of bounded functions of $\dot{B}_{p, \infty}^{1 / p}(\mathbb{R}) \backslash U_{p}(\mathbb{R})$. Also, we do not know whether $\dot{B}_{p, \infty}^{1 / p}(\mathbb{R}) \backslash U_{p}(\mathbb{R})$ contains functions with compact support.

\subsection{Fatou property}

Proposition 14 Let $s \in] 0,2], p, q \in[1,+\infty]$. Let $f$ be a distribution in $\mathbb{R}^{n}$. If $\left(f_{k}\right)_{k \in \mathbb{N}}$ is a sequence in $\dot{B}_{p, q}^{s}\left(\mathbb{R}^{n}\right)$ such that $\left\|f_{k}\right\|_{\dot{B}_{p, q}^{s}\left(\mathbb{R}^{n}\right)} \leq 1$ for all $k$, and such that $\lim _{k \rightarrow+\infty} f_{k}=f$ in the sense of distributions, then $f \in \dot{B}_{p, q}^{s}\left(\mathbb{R}^{n}\right)$ and $\|f\|_{\dot{B}_{p, q}^{s}\left(\mathbb{R}^{n}\right)} \leq 1$.

Proof. The proof given by Franke [15] in case of nonhomogeneous Besov spaces can be easily extended to hold for homogeneous spaces.

\section{References}

[1] Appell, J. And Zabrejko, P.P.: Nonlinear superposition operators. Cambridge Tracts in Mathematics 95. Cambridge Univ. Press, Cambridge, 1990.

[2] Bennett, C. and Sharpley, R.: Interpolation of operators. Pure and Applied Mathematics 129. Academic Press, Boston, 1988.

[3] Bergh, J. and Löfström, J.: Interpolation spaces. An introduction. Grundlehren der Mathematischen Wissenschaften 223. Springer-Verlag, Berlin-New York, 1976.

[4] Bourdaud, G.: Réalisations des espaces de Besov homogènes. Ark. Mat. 26 (1988), no. 1, 41-54.

[5] Bourdaud, G.: Le calcul fonctionnel dans les espaces de Sobolev. Invent. Math. 104 (1991), no. 2, 435-446.

[6] Bourdaud, G.: The functional calculus in Sobolev spaces. In Function spaces, differential operators and nonlinear analysis (Friedrichroda, 1992), 127-142. Teubner-Texte Math. 133. Teubner, Stuttgart, 1993. 
[7] Bourdaud, G.: A sharpness result for powers of Besov functions. J. Funct. Spaces Appl. 2 (2004), no. 3, 267-277.

[8] Bourdaud, G. And Kateb, M. E. D.: Fonctions qui opèrent sur les espaces de Besov. Math. Ann. 303 (1995), no. 4, 653-675.

[9] Bourdaud, G. And Meyer, Y.: Fonctions qui opèrent sur les espaces de Sobolev. J. Funct. Anal. 97 (1991), no. 2, 351-360.

[10] Bourdaud, G. And Meyer, Y.: Le calcul fonctionnel sous-linéaire dans les espaces de Besov homogènes. Rev. Mat. Iberoamericana 22 (2006), no. 2, 725-746.

[11] Bruneau, M.: Variation Totale d'une Fonction. Lecture Notes in Mathematics 413. Springer, Berlin, 1974.

[12] DeVore, R. And Lorentz, G. G.: Constructive Approximation. Grundlehren der mathematischen Wissenschaften. Springer, Berlin, 1993.

[13] Farkas, W. and Leopold, H.-G.: Characterizations of function spaces of generalised smoothness. Ann. Mat. Pura Appl. 185 (2006), no. 1, 1-62.

[14] Federer, H.: Geometric measure theory. Classics in Mathematics. Springer, Berlin, 1969.

[15] Franke, J.: On the space $F_{p, q}^{s}$ of Triebel-Lizorkin type: Pointwise multipliers and spaces on domains. Math. Nachr. 125 (1986), 29-68.

[16] Frazier, M. And Jawerth, B.: Decomposition of Besov Spaces. Indiana Univ. Math. J. 34 (1985), 777-799.

[17] Gol'dman, M. L.: Imbedding theorems for anisotropic Nikol'skij-Besov spaces with moduli of smoothness of general type. Trudy Mat. Inst. Steklov 170 (1984), 86-104.

[18] Giusti, E.: Minimal surfaces and functions of bounded variation. Birkhäuser, Basel, 1984.

[19] Kateb, D.: Fonctions qui opèrent sur les espaces de Besov. Proc. Amer. Math. Soc. 128 (2000), no. 3, 735-743.

[20] Marcinkiewicz, J.: On a class of functions and their Fourier series. C.R. Soc. Sci. Varsovie 26 (1934), 71-77.

[21] Marcus, M. and Mizel, V. J.: Every superposition operator mapping one Sobolev space into another is continuous. J. Funct. Anal. 33 (1979), 217-229.

[22] Oswald, P.: On the boundedness of the mapping $f \rightarrow|f|$ in Besov spaces. Comment. Math. Univ. Carol. 33 (1992), no. 1, 57-66.

[23] Peetre, J.: Interpolation of Lipschitz operators and metric spaces. Mathematica (Cluj) 12 (1970), 325-334.

[24] Peetre, J.: New thoughts on Besov spaces. Duke University Mathematics Series I. Duke University Mathematics Department, Durham, N.C., 1976.

[25] Runst, T. And Sickel, W.: Sobolev spaces of fractional order, Nemytskij operators and nonlinear partial differential equations. De Gruyter Series in Nonlinear Analysis and Applications 3. Walter de Gruyter, Berlin, 1996. 
[26] Savaré, G.: On the regularity of the positive part of functions. Nonlinear Anal. Theory Methods Appl. 27 (1996), no. 9, 1055-1074.

[27] Savaré, G. And Tomarelli, F.: Superposition and chain rule for bounded Hessian functions. Adv. Math. 140 (1998), no. 2, 237-281.

[28] Tartar, L.: Remarks on some interpolation spaces. In Boundary Value Problems for PDEs and Applications, 229-252. Masson, Paris, 1993.

[29] Triebel, H.: Interpolation Theory, Function Spaces, Differential Operators. North-Holland, Amsterdam, 1978.

[30] Triebel, H.: Theory of Function Spaces. Monographs in Mathematics 78. Birkhäuser, Basel, 1983.

[31] Triebel, H.: Theory of Function Spaces II. Monographs in Mathematics 84. Birkhäuser, Basel, 1992.

[32] Wiener, N.: The quadratic variation of a function and its Fourier coefficients. J. Math. Phys. 3 (1924), 72-94.

Recibido: 23 de febrero de 2004.

Gérard Bourdaud

Institut de Mathématiques de Jussieu

Projet d'analyse fonctionnelle

Case 186, 4 place Jussieu, 75252 Paris Cedex 05, France.

bourdaud@ccr.jussieu.fr

Massimo Lanza de Cristoforis Dipartimento di Matematica Pura ed Applicata Università di Padova Via Belzoni 7, 35131 Padova, Italia. mldc@math.unipd.it

Winfried Sickel Mathematisches Institut FSU Jena Ernst-Abbe-Platz 1-2, 07743 Jena, Germany. sickel@minet.uni-jena.de 